TRANSACTIONS OF THE

AMERICAN MATHEMATICAL SOCIETY

Volume 364, Number 9, September 2012, Pages 4465-4494

S 0002-9947(2012)05416-X

Article electronically published on April 18, 2012

\title{
COMPARING THEOREMS OF HYPERARITHMETIC ANALYSIS WITH THE ARITHMETIC BOLZANO-WEIERSTRASS THEOREM
}

\author{
CHRIS J. CONIDIS
}

\begin{abstract}
In $1975 \mathrm{H}$. Friedman introduced two statements of hyperarithmetic analysis, $\mathrm{SL}_{0}$ (sequential limit system) and $\mathrm{ABW}_{0}$ (arithmetic BolzanoWeierstrass), which are motivated by standard and well-known theorems from analysis such as the Bolzano-Weierstrass theorem for $F_{\sigma}$ and $G_{\delta}$ sets of reals. In this article we characterize the reverse mathematical strength of $A B W_{0}$ by comparing it to most known theories of hyperarithmetic analysis.

In particular we show that, over $\mathrm{RCA}_{0}+\mathrm{I} \Sigma_{1}^{1}, \mathrm{SL}_{0}$ is equivalent to $\Sigma_{1}^{1}-\mathrm{AC}_{0}$, and that $A B W_{0}$ is implied by $\Sigma_{1}^{1}-A C_{0}$, and implies weak $-\Sigma_{1}^{1}-A C_{0}$. We then use Steel's method of forcing with tagged trees to show that $A B W_{0}$ is incomparable with INDEC (i.e. Jullien's Theorem) and $\Delta_{1}^{1}-\mathrm{CA}_{0}$. This makes $A B W_{0}$ the first theory of hyperarithmetic analysis that is known to be incomparable with other (known) theories of hyperarithmetic analysis. We also examine the reverse mathematical strength of the Bolzano-Weierstrass theorem in the context of open, closed, $F_{\sigma}, G_{\delta}$, and other types of sets.
\end{abstract}

\section{INTRODUCTION}

The main goal of this paper is to examine the reverse mathematical strength of two statements of second order arithmetic first introduced by Friedman in [Fri75, and motivated by standard, well-known theorems from mathematical analysis such as the Bolzano-Weierstrass theorem for $F_{\sigma}$ and $G_{\delta}$ sets of real numbers (for a general reference on the ongoing program of reverse mathematics, see $\underline{\operatorname{Sim}}$ ). Generally speaking, given a theorem from ordinary mathematics, $T$, the program of reverse mathematics attempts to assign a strength to $T$ based upon the weakest subsystem of second order arithmetic that proves $T$. Very frequently the answer to this question is one of the following five subsystems of second order arithmetic: $\mathrm{RCA}_{0}$ (recursive comprehension axiom), $\mathrm{WKL}_{0}$ (weak König's Lemma), $\mathrm{ACA}_{0}$ (arithmetic comprehension axiom), $\mathrm{ATR}_{0}$ (arithmetic transfinite recursion axiom), and $\Pi_{1}^{1}-\mathrm{CA}_{0}\left(\Pi_{1}^{1}\right.$ comprehension axiom); the system $\mathrm{RCA} \mathrm{A}_{0}$ is almost always assumed. In other words, when one makes a reverse mathematical assertion, one usually means that the assertion holds under the blanket assumption of RCA .

Generally speaking, $\mathrm{RCA}_{0}$ (i.e. recursive comprehension) resembles computable mathematics, and $T$ is equivalent to RCA $\mathrm{R}_{0}$, over $\mathrm{RCA}_{0}$, if there is a proof of $T$ that involves only computable constructions (i.e. the proof can be carried out computably). More specifically, $\mathrm{RCA}_{0}$ says that if a set $A \subseteq \mathbb{N}$ exists, and $B \subseteq \mathbb{N}$ is computable

Received by the editors September 18, 2009 and, in revised form, July 7, 2010.

2010 Mathematics Subject Classification. Primary 03F35; Secondary 03D80.

The author was partially supported by NSERC grant PGS D2-344244-2007. Furthermore, he would like to acknowledge the helpful input he received from his thesis advisors: R.I. Soare, D.R. Hirschfeldt, and A. Montalbán.

(C)2012 American Mathematical Society 4465

Reverts to public domain 28 years from publication 
from $A$ (i.e. $B$ is Turing reducible to $A$ ), written $B \leq_{T} A$, then $B$ also exists. Note that, if we take $A=\emptyset$, then we get that $\mathrm{RCA}_{0}$ implies that the computable sets exist. Also, one can show that the $\omega$-models that satisfy $\mathrm{RCA}_{0}$ are exactly those that are closed downwards under $\leq_{T}$ and closed under disjoint union. Here the disjoint union of two sets $A, B \subseteq \mathbb{N}$ is equal to the set $\{2 n: n \in A\} \cup\{2 n+1: n \in B\}$. By $\omega$-model, we mean a model of second order arithmetic whose first order part is equal to the standard natural numbers $(\omega, 0,1,+, \times)$. Such models are normally identified by their second order parts.

The behavior of $\mathrm{ACA}_{0}$ (arithmetic comprehension) is similar to that of $\mathrm{RCA}_{0}$, but with respect to arithmetic reducibility instead of Turing reducibility. A set $B \subseteq \mathbb{N}$ is arithmetically reducible to a set $A \subseteq \mathbb{N}$ if there exists $n \in \mathbb{N}$ such that $B$ is computable from $A^{(n)}$ (here $A^{(n)}$ denotes the $n^{\text {th }}$ Turing jump of $A$ ), or, equivalently, if $B$ is definable by an arithmetic formula with $A$ as a parameter (informally, a formula is arithmetic if it has finitely many number quantifiers and no set quantifiers; a more formal definition is given in the Basic notation and background subsection at the end of this section). An $\omega$-model is a model of ACA $_{0}$ if and only if it is closed under arithmetic reducibility and disjoint union. In this case we have that $\mathrm{ACA}_{0}$ implies $T$ if there is a proof of $T$ that involves only arithmetical constructions. It is well known that $\mathrm{ACA}_{0}$ implies $\mathrm{WKL}_{0}$, the theory of second order arithmetic which says that $\mathrm{RCA}_{0}$ holds and that every infinite binary branching tree has an infinite path. This fact will be used in the proof of Theorem 2.1. Next, we define the class of hyperarithmetic sets, a main focus of our study.

The main purpose of this article is to determine the reverse mathematical strength of various standard, well-known theorems of mathematical analysis all of which are motivated by compactness and the Bolzano-Weierstrass theorem for sets of different complexities (such as $F_{\sigma}$ and $G_{\delta}$ sets of real numbers). Two of these statements were first introduced by H. Friedman in [Fri75]. One of Friedman's statements is denoted by $\mathrm{SL}_{0}$ (sequential limit system), and says that if $A(X)$ is an arithmetic predicate of reals such that $\{X: A(X)\}$ has an accumulation point, $Z_{0}$, then there is an infinite subsequence of $\{X: A(X)\}$ that converges to $Z_{0}$. The second statement is denoted by $\mathrm{ABW}_{0}$ (arithmetic Bolzano-Weierstrass), and says that if $A(X)$ is a bounded arithmetical predicate of reals, then $A(X)$ either has finitely many solutions, or else $\{X: A(X)\}$ has an accumulation point $Z_{0}\left(Z_{0}\right.$ need not satisfy $A(X)$ ). We also examine the restriction of $\mathrm{ABW}_{0}$ to arithmetic predicates of various low complexities corresponding to open, closed, $F_{\sigma}, G_{\delta}$, and other types of sets of real numbers and show that these theorems quickly become much stronger than arithmetic comprehension. Furthermore, both of our main theorems about $A B W_{0}$ yield corollaries pertaining to certain restrictions of $A B W_{0}$ as described in the previous sentence. More information on hyperarithmetic sets and hyperarithmetic reducibility is given in the next section (i.e. Section 1.1). More information on theories of hyperarithmetic analysis is given in Section 1.2. For more information on $\mathrm{SL}_{0}$ and $\mathrm{ABW}$, consult Section 1.3.

We now (briefly) state our main results. In the next section (i.e. Section 2), we prove that, over $R C A_{0}+I \Sigma_{1}^{1}, S L_{0}$ is equivalent to $\Sigma_{1}^{1}-A C_{0}$, and that $A B W_{0}$ is implied by $\Sigma_{1}^{1}-A C_{0}$, and implies weak $-\Sigma_{1}^{1}-A C_{0}$. We also analyze the strengths of various restricted versions of $\mathrm{ABW}_{0}$ (to be defined in Section 1.4 below). Then, in Sections 3 and 4, we employ Steel's method of forcing with tagged trees Ste78. to prove our main theorems which (when taken together) imply that $A B W_{0}$ is 
incomparable with other theories of hyperarithmetic analysis called INDEC (i.e. Jullien's Theorem) and $\Delta_{1}^{1}-\mathrm{AC}_{0}$ (see Section 1.2 for more information on theories of hyperarithmetic analysis). In addition, we will obtain various corollaries that shed light on the reverse mathematical strength of the restrictions of $\mathrm{ABW}_{0}$ that we briefly mentioned in the previous paragraph. A more precise description of the information in this paragraph is given in Section 1.4.

1.1. Hyperarithmetic sets. Let $\mathcal{L}=\left\langle L, \leq_{L}\right\rangle, L \subset \omega$, be a presentation of a linear ordering which has least element $0 \in \omega$. Given sets $X, Y \subseteq \omega$, we say that $Y$ is an $H(X, \mathcal{L})$-set if $Y^{[0]}=X$, and for every $l \in L \backslash\{0\}$ we have that

$$
Y^{[l]}=\bigoplus_{k<L_{L} l}\left(Y^{[k]}\right)^{\prime}
$$

where $Y^{[j]}=\{n \in \omega:\langle j, n\rangle \in Y\}$ and $\bigoplus_{k \in A} B_{k}=\left\{\langle k, n\rangle: k \in A, n \in B_{k}\right\}$ (here $\langle\cdot, \cdot\rangle: \omega^{2} \rightarrow \omega$ is a computable pairing function, as defined in Soa]).

It is not difficult to show that, when $\mathcal{L}$ is an ordinal (i.e. $\mathcal{L}$ has no infinite descending sequences), then there exists a unique $H(X, \mathcal{L})$-set, which we denote by $X^{(\mathcal{L})}$. However, if we let $\mathcal{L}^{\prime}$ be a different presentation of an isomorphic copy of $\mathcal{L}$, then the Turing degree of $X^{\left(\mathcal{L}^{\prime}\right)}$ may not equal that of $X^{(\mathcal{L})}$. But, if we take $\mathcal{L}$ to be a computable ordinal (as defined in [AK]), then, by a result of Spector [Spe55], all $H(X, \mathcal{L})$ sets are Turing equivalent (where $\mathcal{L}$ ranges over different computable presentations of a fixed computable ordinal). We denote the least non- $X$-computable ordinal by $\omega_{1}^{X}$, and write $\omega_{1}^{C K}$ for $\omega_{1}^{\emptyset}$ (where $C K$ stands for Church-Kleene). It is well known that the set of computable ordinals is closed downwards $\mathrm{AK}$.

A formula $\varphi$ of second order arithmetic is $\Sigma_{1}^{1}$ (relative to $A \subseteq \omega$ ) if it is of the form $(\exists X) \psi$, for some arithmetic formula $\psi$ (with $A$ as a parameter), and $X$ is a set variable. A set $B \subseteq \omega$ is $\Sigma_{1}^{1}$ (relative to $A \subseteq \omega$ ), written $B \in \Sigma_{1}^{1}\left(B \in \Sigma_{1}^{1}(A)\right.$ ), if it is definable by a $\Sigma_{1}^{1}$ formula (with parameters from $A$ ). We say that $B \subseteq \omega$ is $\Delta_{1}^{1}$ (relative to $\left.A \subseteq \omega\right)$, and write $B \in \Delta_{1}^{1}\left(B \in \Delta_{1}^{1}(A)\right)$, if there exist $\Sigma_{1}^{1}$ formulas $\varphi, \psi$ (with $A$ as a parameter) such that

$$
(\forall n)[(n \in B) \Leftrightarrow(\varphi(n) \Leftrightarrow \neg \psi(n))] .
$$

Theorem 1.1 ([AK] $)$. For any two sets $A, B \subseteq \omega$, the following are equivalent:

(1) $\left(\exists \alpha<\omega_{1}^{A}\right) B \leq_{T} A^{(\alpha)}$.

(2) $B \in \Delta_{1}^{1}(A)$.

(3) There is an A-computable infinitary formula $\varphi$ such that $X=\{n$ : $\varphi(n)\}$.

If $\omega_{1}^{A}=\omega_{1}^{C K}$, then we also have that:

(4) there is a computable infinitary formula $\varphi$ such that $X=\{n: \varphi(n, Y)\}$.

A brief explanation of computably infinitary formulas is given at the end of this section (for more information, see the subsection called Basic notation and background).

Definition 1.2. Whenever $A, B \subseteq \omega$ satisfy any of conditions (1)-(3) above, we say that $B$ is hyperarithmetically reducible to $A$, and write $B \leq_{H} A$. We also define $\operatorname{HYP}(A)=\left\{X \subseteq \omega: X \leq_{H} A\right\}$ and $\operatorname{HYP}=\operatorname{HYP}(\emptyset)$.

More information on hyperarithmetic sets and hyperarithmetic reducibility can be found in $\mathrm{AK}$. 


\subsection{Theories of hyperarithmetic analysis.}

Definition 1.3. Let $T$ be a collection of axioms of second order arithmetic. We say that $T$ is a theory of hyperarithmetic analysis if

(1) $T$ holds in $\operatorname{HYP}(A)$, for every $A \subseteq \omega$, where $\operatorname{HYP}(A)$ is the $\omega$-model consisting of the sets that are hyperarithmetically reducible to $A$.

(2) All $\omega$-models of $T$ are hyperarithmetically closed.

This is equivalent to saying that, for every $A \subseteq \omega, \operatorname{HYP}(A)$ is the minimum $\omega$ model of $T$ that contains $A$, and that every $\omega$-model of $T$ is closed under disjoint unions.

Thus, the theories of hyperarithmetic analysis are those axioms of second order arithmetic that characterize both HYP and hyperarithmetic reduction. They also seem to exhibit a unique and interesting behavior in that small modifications often yield inequivalent theories. In the next section we will use the fact that all the theories of hyperarithmetic analysis mentioned in this section (and, indeed, this article) imply $\mathrm{ACA}_{0}$. This fact is already known (see, for example, Mon06]) for those theories of hyperarithmetic analysis that are not equal to $A B W_{0}$ and $S_{0}$, which will be defined below in the next subsection (i.e. Section 1.3). We will prove this fact in the case of $\mathrm{SL}_{0}$ and $A B W_{0} \sqrt{1}$ in Section 2 (Lemma 2.2).

Definition 1.4. A sentence $S$ is a sentence (statement, theorem) of hyperarithmetic analysis if $\mathrm{RCA}_{0}+S$ is a theory of hyperarithmetic analysis.

Before we can state the well-known statements of hyperarithmetic analysis that will play a central role in this article, we require a definition and a theorem of Jullien JJul. Jullien's Theorem was first examined in the context of reverse mathematics by Montalbán in Mon06.

Definition 1.5. Given a linear ordering $\mathcal{Z}=\langle Z, \leq\rangle$, we define a cut in $\mathcal{Z}$ to be a pair of sets $\langle L, R\rangle$ such that $L=Z \backslash R$ is an initial segment of $\mathcal{Z}$. We say that $\mathcal{Z}$ is indecomposable if, for every cut $\langle L, R\rangle, \mathcal{Z}$ embeds into either $L$, or else $\mathcal{Z}$ embeds into $R$ (thinking of $L$ and $R$ as suborderings of $\mathcal{Z}$ ). We say that $\mathcal{Z}$ is indecomposable to the right if, for every cut $\langle L, R\rangle$ with $R \neq \emptyset, \mathcal{Z}$ embeds into $R$. We define indecomposable to the left in a similar fashion. A linear order is called scattered if $\eta$, the order type of the rational numbers, does not embed in it.

Theorem 1.6 (Jullien's Theorem, Jul]). Every scattered indecomposable linear order is either indecomposable to the right, or indecomposable to the left.

We say that a computable infinitary formula (see Section 1.5 .2 for more details) $\varphi$ is determined if there is a map $v: \operatorname{subformulas}(\varphi) \rightarrow\{$ True, False $\}$ such that the obvious logical rules hold.

The following list consists of the well-known theories of hyperarithmetic analysis that play a significant role in this article.

$\left(\Sigma_{1}^{1}-\mathrm{AC}_{0}\right)$ If $A(X, n)$ is an arithmetic predicate with a free set variable $X$ and a free number variable $n$, then we have

$$
(\forall n)(\exists Y)\left[A(Y, n) \Rightarrow(\exists Z)(\forall n)\left[A\left(Z^{[n]}, n\right)\right]\right] .
$$

\footnotetext{
${ }^{1}$ To show that $A B W_{0}$ implies $A C A_{0}$ we require the additional hypothesis of induction for $\Sigma_{1}^{1}$ formulas, which we denote by $I \Sigma_{1}^{1}$, and which we define in Section 1.5.
} 
$\left(\Pi_{1}^{1}-\mathrm{SEP}_{0}\right)$ If $\varphi, \psi$ are $\Sigma_{1}^{1}$ formulas satisfying $(\forall n)[\neg(\neg \varphi(n) \wedge \neg \psi(n))]$, then there exists a set $D$ such that $(\forall n \in D)[\neg \varphi(n) \wedge \psi(n)]$.

$\left(\Delta_{1}^{1}-\mathrm{CA}_{0}\right)$ If $\varphi, \psi$ are $\Sigma_{1}^{1}$ formulas such that $(\forall n)[\varphi(n) \vee \psi(n)]$ and $(\forall n)[\neg \varphi(n) \Leftrightarrow$ $\psi(n)]$, then there exists a set $D$ such that $(\forall n)[n \in D \Leftrightarrow \varphi(n)]$.

$\left(\mathrm{INDEC}_{0}\right)$ Every scattered indecomposable linear order is either indecomposable to the right or indecomposable to the left. (Jullien's Theorem)

$\left(\Sigma_{1}^{1}-\mathrm{AC}_{0}^{\mathrm{w}}\right)$ If $A(X, n)$ is an arithmetic predicate with a single free set variable $X$ and a single free number variable $n$, then we have

$$
(\forall n)(\exists ! Y)\left[A(Y, n) \Rightarrow(\exists Z)(\forall n)\left[A\left(Z^{[n]}, n\right)\right]\right] .
$$

$\left(\mathrm{L}_{\omega_{1}, \omega} \mathrm{CA}_{0}\right)$ Let $\left\{\varphi_{n}\right\}$ be a sequence of determined computable infinitary sentences. Then there exists a set $D$ such that $n \in D \Leftrightarrow \varphi_{n}$ is true.

Throughout this article we shall denote the weak $\Sigma_{1}^{1}$ choice scheme by $\Sigma_{1}^{1}-\mathrm{AC}_{0}^{\mathrm{w}}$ (as above), or weak $-\Sigma_{1}^{1}-\mathrm{AC}_{0}$. We prefer to use the latter, but use the former at certain times (as above) because it is more compact.

Montalbán (unpublished) has recently shown that $\mathrm{L}_{\omega_{1}, \omega} \mathrm{CA}_{0}$ (above) is a theorem of hyperarithmetic analysis that is implied by the restriction of weak $-\Sigma_{1}^{1}-\mathrm{AC}_{0}$ to two-quantifier arithmetic predicates of the form $\forall \exists \ldots 2$. For more information on computably infinitary formulas, consult Section 1.5 .2 below.

The statements $\Sigma_{1}^{1}-A C_{0}$ and $\Delta_{1}^{1}-C_{0}$ were first introduced by Kreisel in Kre62, along with another statement of hyperarithmetic analysis known as $\Sigma_{1}^{1}$ dependent choice. Kreisel also asked whether or not $\Sigma_{1}^{1}-\mathrm{AC}_{0}$ implies $\Sigma_{1}^{1}$ dependent choice and whether $\Delta_{1}^{1}-\mathrm{CA}_{0}$ implies $\Sigma_{1}^{1}-\mathrm{AC}_{0}$ (the opposite implications are both trivial). Soon after that, H. Friedman [Fri] proved that $\Sigma_{1}^{1}$ dependent choice is strictly stronger than $\Sigma_{1}^{1}-\mathrm{AC}_{0}$. Later on, Steel Ste78] showed that $\Sigma_{1}^{1}-\mathrm{AC}_{0}$ is strictly stronger than $\Delta_{1}^{1}-C A_{0}$. Furthermore, over the past forty years, it has been established that (over $\mathrm{RCA}_{0}$ )

$$
\left(\Sigma_{1}^{1}-\mathrm{AC}_{0}\right) \rightarrow\left(\Pi_{1}^{1}-\mathrm{SEP}_{0}\right) \rightarrow\left(\Delta_{1}^{1}-\mathrm{CA}_{0}\right) \rightarrow\left(\mathrm{INDEC}_{0}\right) \rightarrow\left(\Sigma_{1}^{1}-\mathrm{AC}_{0}^{\mathrm{w}}\right) \rightarrow\left(\mathrm{L}_{\omega_{1}, \omega} \mathrm{CA}_{0}\right),
$$

and none of the arrows is reversible, except possibly the rightmost. Each of the above implications is straightforward, except for $\Delta_{1}^{1}-\mathrm{CA}_{0} \rightarrow$ INDEC $_{0}$ and weak $-\Sigma_{1}^{1}-\mathrm{AC}_{0} \rightarrow \mathrm{L}_{\omega_{1}, \omega} \mathrm{CA}_{0}$, proven by Montalbán in [Mon06, and INDEC $\mathrm{C}_{0} \rightarrow$ weak $-\Sigma_{1}^{1}-A C_{0}$, shown by Neeman in Nee. On the other hand, the fact that the two leftmost arrows cannot be reversed was first established by Montalbán in [Mon06, Mon], while the irreversibility of the next two (leftmost) arrows was established by Neeman in Nee. It should also be noted that Steel Ste78] first developed the main technique of this article (i.e. forcing with tagged trees) to prove that $\Delta_{1}^{1}-\mathrm{CA}_{0}$ does not imply $\Sigma_{1}^{1}-\mathrm{AC}_{0}$, while van Wesep [vW] subsequently used a variant of Steel's technique to show that weak $-\Sigma_{1}^{1}-A C_{0}$ does not imply $\Delta_{1}^{1}-A C_{0}$.

1.3. $\mathrm{SL}_{0}, A B W_{0}$, and restrictions of $A B W_{0}$. We now introduce the two statements of hyperarithmetic analysis, $\mathrm{SL}_{0}$ (sequential limit system) and $\mathrm{ABW}_{0}$ (arithmetic Bolzano-Weierstrass), that are the main focus of our study in this paper. $\mathrm{SL}_{0}$ and $A B W_{0}$ were first introduced by Friedman in [Fri75], and come from basic, wellknown theorems in mathematical analysis. Our statement of $A B W_{0}$ differs slightly

\footnotetext{
${ }^{2}$ In fact, Montalbán has shown that $\mathrm{L}_{\omega_{1}, \omega} \mathrm{CA}_{0}$ is equivalent to a theorem of hyperarithmetic analysis known as CDG - CA (see Mon06] for more details including the definition of CDG - CA). In [Mon06, page 113], Montalbán proves that the restriction of weak $-\Sigma_{1}^{1}-\mathrm{AC}_{0}$ to two-quantifier arithmetic predicates of the form $\forall \exists \cdots$ implies CDG - CA.
} 
from that of Friedman [Fri75, which mentions predicates of real numbers. We now state Friedman's version of ABW.

(ABW) Consists of RCA (i.e. RCA $A_{0}$, plus the induction scheme for all formulas) together with the axioms which assert that to every bounded arithmetic predicate of reals there is either a finite sequence of reals that includes all solutions, or a real, every neighborhood of which contains at least two solutions.

It is easy to prove that, over $R C A_{0}$, our version of $A B W_{0}$ (below) is equivalent to that of Friedman (above) minus the induction scheme for all formulas. Although we prefer to think of $A B W_{0}$ in terms of Friedman's definition above, we find our version of $A B W_{0}$ (below) to be more convenient for carrying out proofs related to $A B W_{0}$, and therefore after this section we will work exclusively with our version of $A B W_{0}$, given below.

Let $A(X)$ be an arithmetic predicate (possibly with parameters) of reals with a single free set (i.e. real) variable $X$ and no other free variables. Let $Z$ be a set of reals. Recall that a real $Z_{0}$ is said to be an accumulation point of the set $Z$ if every neighborhood of $Z_{0}$ contains some $X \in Z$ such that $X \neq Z_{0}$ ( $Z_{0}$ need not belong to $Z$ ). In other words, $Z_{0}$ is an accumulation point of $Z$ if

$$
(\forall n)(\exists X \in Z)\left[( X \neq Z _ { 0 } ) \wedge \left(X\left\lceil n=Z_{0}\lceil n)\right] .\right.\right.
$$

Note that this is equivalent to saying that every neighborhood of $Z_{0}$ contains at least two elements of $Z$. We say that a predicate is bounded if its solutions all live in Cantor space ${ }^{3}$ (we define Cantor space in the next subsection, called Basic notation and background). Over $\mathrm{RCA}_{0}$, our definition of bounded predicate is equivalent to many other standard definitions.

$\left(\mathrm{SL}_{0}\right)$ If $A(X)$ has an accumulation point $Z_{0}$, then there is an infinite sequence of reals, $\left\{X_{n}\right\}_{n \in \mathbb{N}}$, such that $(\forall n)\left[A\left(X_{n}\right)\right]$ and $\lim _{n} X_{n}=Z_{0}$.

$\left(\mathrm{ABW}_{0}\right)$ If $A(X)$ is bounded, then either $A(X)$ has finitely many solutions, or else the set of solutions to $A,\{X: A(X)\}$, has an accumulation point.

It should be noted that Friedman originally introduced $S_{0}$ and $A B W_{0}$ in the context of unrestricted induction, and in doing so did not include the subscript 04

The following are various restrictions of $\mathrm{ABW}_{0}$ that are perhaps more natural than $\mathrm{ABW}_{0}$ because they do not make use of arithmetic predicates, a notion that is not used outside of logic.

$\left(\mathrm{OABW} \mathrm{W}_{0}\right)$ Every bounded open set of reals has either finitely many points or an accumulation point.

$\left(C A B W_{0}\right)$ Every bounded closed set of reals has either finitely many points or an accumulation point.

$\left(\mathrm{F}_{\sigma} \mathrm{ABW}_{0}\right)$ Every bounded $F_{\sigma}$ set of reals has either finitely many points or an accumulation point.

$\left(\mathrm{G}_{\delta} \mathrm{ABW}_{0}\right)$ Every bounded $G_{\delta}$ set of reals has either finitely many points or an accumulation point.

\footnotetext{
${ }^{3}$ It is not difficult to show that, over $\mathrm{RCA}_{0}$, this is equivalent to the standard definition of a bounded set of reals.

${ }^{4} \mathrm{By}$ including the subscript 0 in $\mathrm{SL}_{0}$ and $\mathrm{ABW}_{0}$, we indicate that we are only assuming induction for $\Sigma_{1}^{0}$ formulas. This convention applies to all subsystems of second order arithmetic (i.e. $R C A_{0}, W K L_{0}, A C A_{0}$, etc.).
} 
$\left(\mathrm{F}_{\sigma \delta} \mathrm{ABW}_{0}\right)$ Every bounded set that is an intersection of $F_{\sigma}$ sets of reals has either finitely many points or an accumulation point.

$\left(\mathrm{G}_{\delta \sigma} \mathrm{ABW}_{0}\right)$ Every bounded set that is a union of $G_{\delta}$ sets of reals has either finitely many points or an accumulation point.

The notation $F_{\sigma \delta}$ and $G_{\delta \sigma}$ will be explained in Section 1.5 .2 below. In the next section we will show that $O A B W_{0}$ is equivalent to $\mathrm{RCA}_{0} ; \mathrm{CABW}_{0}$ and $\mathrm{F}_{\sigma} \mathrm{ABW}_{0}$ are equivalent to $A C A_{0} ; G_{\delta} A B W_{0}$ and $F_{\sigma \delta} A B W_{0}$ imply $A C A_{0}$; and $G_{\delta \sigma} A B W_{0}$ is a statement of hyperarithmetic analysis. Furthermore, our main theorems in Sections 3 and 4 will show that, like $A B W_{0}$, we have that $G_{\delta} A_{B W}, F_{\sigma \delta} A B W_{0}$, and $G_{\delta \sigma} A B B W_{0}$ are incomparable with other theorems of hyperarithmetic analysis (in particular INDEC 0 and $\Delta_{1}^{1}-\mathrm{CA}_{0}$; see Corollaries 3.18 and 4.2 below for more details). As a corollary we will conclude that $\mathrm{G}_{\delta} \mathrm{ABW}_{0}$ and $\mathrm{F}_{\sigma \delta} \mathrm{ABW}_{0}$ are strictly stronger than $\mathrm{ACA}_{0}$ (Corollary 3.19). We do not know whether or not $\mathrm{G}_{\delta} \mathrm{ABW}_{0}$ and $\mathrm{F}_{\sigma \delta} \mathrm{ABW}_{0}$ are statements of hyperarithmetic analysis.

Question 1.7. Are $\mathrm{G}_{\delta} \mathrm{ABW}_{0}$ and $\mathrm{F}_{\sigma \delta} \mathrm{ABW}_{0}$ statements of hyperarithmetic analysis?

1.4. Our main results. Friedman's article Fri75 serves as our starting point. In Fri75, Friedman makes the following assertions.

Proposition 1.8 ([Fri75, Theorem 2.1]). Over $\mathrm{RCA}, \mathrm{SL}_{0}$ is equivalent to $\Sigma_{1}^{1}-\mathrm{AC}_{0}$. In other words, if we assume $\mathrm{RCA}_{0}$ and induction for all formulas of second order arithmetic, then $\mathrm{SL}_{0}$ is equivalent to $\Sigma_{1}^{1}-\mathrm{AC}_{0}$.

Proposition 1.9 ([Fri75, page 239]). Over RCA, we have that $\Sigma_{1}^{1}-\mathrm{AC}_{0}$ implies $\mathrm{ABW}_{0}$.

One of our main goals in the next section (Theorem 2.1) is to provide explicit proofs of the previous two propositions. To the author's knowledge, no proof of either proposition has ever been published, but the author believes that Friedman must have had proofs similar to the ones given in the next section before [Fri75.

In the next section (Theorem 2.1) we prove the following theorem.

Theorem 2.1 ([Fri75, page 239]). Over $\mathrm{RCA}_{0}+\mathrm{I} \Sigma_{1}^{1}$ (here $\mathrm{I} \Sigma_{1}^{1}$ denotes $\Sigma_{1}^{1}$-induction and is defined in the next subsection), $\mathrm{SL}_{0}$ is equivalent to $\Sigma_{1}^{1}-\mathrm{AC}_{0}$, while $\mathrm{ABW}_{0}$ implies weak $-\Sigma_{1}^{1}-\mathrm{AC}_{0}$ and is implied by $\Sigma_{1}^{1}-\mathrm{AC}_{0}$. It follows that $\mathrm{SL}_{0}$ and $\mathrm{ABW}_{0}$ are statements of hyperarithmetic analysis.

As a corollary we will be able to conclude that $\mathrm{G}_{\delta \sigma} \mathrm{ABW}_{0}$ (above) is a theorem of hyperarithmetic analysis. In Theorem 2.4 we will analyze the reverse mathematical strengths of $\mathrm{OABW}_{0}, \mathrm{CABW}_{0}, \mathrm{~F}_{\sigma} \mathrm{ABW}_{0}, \mathrm{G}_{\delta} \mathrm{ABW}_{0}$, and $\mathrm{F}_{\sigma \delta} \mathrm{ABW}_{0}$ (see the end of the previous subsection for our results).

We do not know whether or not our hypothesis of $I \Sigma_{1}^{1}$ can be reduced or eliminated in Theorem 2.1 above.

Question 1.10. Do the implications of Theorem 2.1 (above) hold over RCA $A_{0}$ ?

In Section 3 we prove the following theorem.

Theorem 3.1. There is an $\omega$-model of $\mathrm{ABW}_{0}$ that is not a model of $\Delta_{1}^{1}-\mathrm{CA}_{0}$. Therefore, $\mathrm{ABW}_{0}$ does not imply $\Delta_{1}^{1}-\mathrm{CA}_{0}$. 
In Section 4 we prove the following theorem.

Theorem 4.1. There is an $\omega$-model of $\mathrm{ABW}_{0}$ that is not a model of INDEC. Therefore, $\mathrm{ABW}_{0}$ does not imply INDEC $\mathrm{I}_{0}$.

These results imply that the reverse mathematical strength of $A B W_{0}$ lies strictly between those of $\Sigma_{1}^{1}-A C_{0}$ and weak $-\Sigma_{1}^{1}-A C_{0}$, and that the strength of $A B W_{0}$ is incomparable to those of $\Delta_{1}^{1}-C_{0}$ and INDEC $C_{0}$. These facts make $A B W_{0}$ the first theory of hyperarithmetic analysis that is known to be incomparable to other theories of hyperarithmetic analysis. As a corollary we will conclude that $\mathrm{G}_{\delta} \mathrm{ABW}_{0}$, $\mathrm{F}_{\sigma \delta} \mathrm{ABW}_{0}$, and $\mathrm{G}_{\delta \sigma} \mathrm{ABW}_{0}$ are also incomparable with both $\Delta_{1}^{1}-\mathrm{CA}_{0}$ and INDEC (see Corollaries 3.18 and 4.2 below). We leave the following question open, and conjecture that its answer is "yes".

Question 1.11. Is every $\omega$-model of $\Pi_{1}^{1}-S E P_{0}$ also a model of $A B W_{0}$ ? In other words, in the context of $\omega$-models, does $\Pi_{1}^{1}-\mathrm{SEP}_{0}$ imply $\mathrm{ABW}_{0}$ ?

Figure 1 summarizes our main results (over $R C A_{0}+I \Sigma_{1}^{1}$ ).

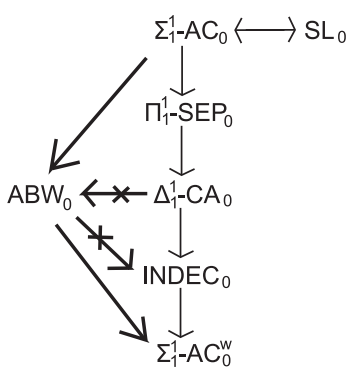

Figure 1

\subsection{Basic notation and background.}

1.5.1. Cantor space and Baire space. We use $2^{\omega}$ to denote Cantor space, the space of infinite sequences of $0 \mathrm{~s}$ and $1 \mathrm{~s}$. We use $2^{<\omega}$ to denote the full binary tree, or the set of finite sequences of $0 \mathrm{~s}$ and $1 \mathrm{~s}$. If $\sigma \in 2^{<\omega}$, and either $\tau \in 2^{<\omega}$ or $\tau \in 2^{\omega}$, we write $\sigma \subseteq \tau$ to mean that $\tau$ extends $\sigma$, or (equivalently) that $\sigma$ is an initial segment of $\tau$. We define $\sigma \subset \tau$ to mean that $\sigma \subseteq \tau$ and $\sigma \neq \tau$. If $X \in 2^{\omega}$ and $n \in \omega$, we write $X\left\lceil n\right.$ to denote the initial segment of $X$ of length $n$. A set $T \subseteq 2^{<\omega}$ is called a tree if $T$ is closed under initial segments, and for any given tree $T \subseteq 2^{<\omega}$ and $f \in 2^{\omega}$, we say that $f$ is a path through $T$ if for every $n \in \omega$ we have that $f \nmid n=\langle f(0), f(1), \ldots, f(n-1)\rangle \in T$. Furthermore, if $T \subseteq 2^{<\omega}$ is a tree, we define $[T]$ to be the set of paths in $2^{\omega}$ through $T$.

The standard topology on $2^{\omega}$ is obtained by defining basic open sets of the form

$$
[\sigma]=\left\{X \in 2^{\omega}: \sigma \subset X\right\}
$$

for every $\sigma \in 2^{<\omega}$. It follows that a sequence of points $\left\{X_{n}: n \in \omega\right\} \subseteq 2^{\omega}$ converges to $X \in 2^{\omega}$ if and only if

$$
(\forall k \in \omega)(\exists m \in \omega)(\forall n \geq m)\left[X_{n}\lceil k=X\lceil k] .\right.
$$

Let $\omega^{\omega}$ denote the set of infinite strings of natural numbers (Baire space), and let $\omega^{<\omega}$ denote the set of finite strings of natural numbers. For all $X \in \omega^{\omega}, \sigma, \tau \in \omega^{<\omega}$, 
and $n \in \omega$, we define $\sigma \subseteq X, \sigma \subseteq \tau, \sigma \subset X, \sigma \subset \tau, X \uparrow n$, tree, path, [T] (where $T \subseteq \omega^{<\omega}$ is a tree), and basic open sets $[\sigma] \subseteq \omega^{\omega}$ analogously to the case of Cantor space.

Given a tree $T \subseteq \omega^{<\omega}$, and $\sigma \in T$, we define the well-founded rank of $\sigma$ (relative to $T$ ), denoted by $|\sigma|_{T}$, such that $|\sigma|_{T}=\sup \left\{|\tau|_{T}+1: \tau \in T, \sigma \subset \tau\right\}$. It follows that $\sigma \in T$ is not in the well-founded part of $T \subseteq \omega^{<\omega}$ exactly when $|\sigma|_{T}=\infty$. Lastly, for any given sets $A, B \subseteq \omega$, we will use the notation $A \subset_{f} B$ to mean that $A$ is a finite subset of $B$.

We will assume that the reader is familiar with the basics of reverse mathematics, as presented in Simpson's book [Sim. We will use $I \Sigma_{1}^{1}\left(\Sigma_{1}^{1}\right.$-induction) to denote the scheme which says that for any $\Sigma_{1}^{1}$ formula $\varphi$ the following holds:

$$
(\varphi(0) \wedge(\forall n)[\varphi(n) \rightarrow \varphi(n+1)]) \rightarrow(\forall n) \varphi(n) .
$$

For the definition of the $\Sigma_{1}^{1}$ formula, or more information on the role of induction in reverse mathematics, consult Sim.

1.5.2. Arithmetical and hyperarithmetical formulas and hierarchies. A formula $\varphi$ is arithmetic if it contains only number quantifiers (i.e. $\varphi$ contains no set quantifiers). More specifically, a formula is $\Sigma_{0}^{0}$ or $\Pi_{0}^{0}$ if it is an open formula (i.e. it contains only bounded quantifiers). Now, for any $n \in \omega$, a formula $\varphi$ is $\Sigma_{n}^{0}, n>0$, if $\varphi$ is of the form $(\exists \bar{x}) \psi(\bar{x})$, and $\psi(\bar{x})$ is $\Pi_{n-1}^{0}$. A formula $\varphi$ is $\Pi_{n}^{0}, n>0$, if $\varphi$ is of the form $(\forall \bar{x}) \psi(\bar{x})$, and $\psi(\bar{x})$ is $\Sigma_{n-1}^{0}$. We can also define the sets of $\Sigma_{n}^{0}\left(A_{0}, A_{1}, \ldots, A_{k}\right)$ and $\Pi_{n}^{0}\left(A_{0}, A_{1}, \ldots, A_{k}\right)$ formulas in the same way, except that we also allow the sets $A_{0}, A_{1}, \ldots, A_{k}$ to be used as parameters in these formulas. A formula is arithmetic (relative to $\left.A_{0}, A_{1}, \ldots, A_{n}\right)$ if it is $\Sigma_{n}^{0}\left(\Sigma_{n}^{0}\left(A_{0}, A_{1}, \ldots, A_{n}\right)\right.$ ), for some $n \in \omega$. A set $A \subseteq \omega$ is arithmetic (relative to $A_{0}, A_{1}, \ldots, A_{k}$ ) if it is definable by an arithmetic formula (with parameters $A_{0}, A_{1}, \ldots, A_{k}$ ). More information on arithmetic formulas and arithmetic sets can be found in Soa.

Let $A \subseteq 2^{\omega}$. We say that $A$ is a $\Sigma_{n}^{0}\left(\right.$ or $\Pi_{n}^{0}$ )-class if there is a $\Sigma_{n}^{0}$ (or $\left.\Pi_{n}^{0}\right)$ formula $\varphi$ such that

$$
A=\left\{X \in 2^{\omega}: \varphi(X)\right\} .
$$

Similarly, one can define the notion of $\Sigma_{n}^{0}(B)$-class and $\Pi_{n}^{0}(B)$-class, for any parameter $B \subset \mathbb{N}$. It is well known that $A$ is $F_{\sigma}$ if and only if there is a set $B \subseteq \omega$ such that $A$ is a $\Sigma_{2}^{0}(B)$-class, and $A$ is $G_{\delta}$ if and only if there is a set $B \subseteq \omega$ such that $A$ is a $\Pi_{2}^{0}(B)$-class. Hence, the $F_{\sigma}$ and $G_{\delta}$ subsets of Cantor space are arithmetically definable (with parameters). We will also say that $A$ is $F_{\sigma \delta}$ whenever there exists a parameter $B$ such that $A$ is a $\Pi_{3}^{0, B}$-class, and similarly $A$ is $G_{\delta \sigma}$ whenever $A$ is $\Sigma_{3}^{0, B}$, for some parameter $B$. This is equivalent to saying that $A$ is an intersection of $F_{\sigma}$ sets, or that $A$ is the union of $G_{\delta}$ sets, respectively. One of the goals of this article is to examine the reverse mathematical strength of the Bolzano-Weierstrass theorem (i.e. $\mathrm{ABW}_{0}$ ) in the context of open, closed, $F_{\sigma}, G_{\delta}, F_{\sigma \delta}$, and $G_{\delta \sigma}$ sets. This is primarily done in the next section.

A set $A \subseteq \omega$ is computably enumerable (c.e.) if it is the range of a 1-1 computable function. In other words, $A$ is c.e. if there is an algorithm that lists the elements of $A$ (not necessarily in order). Generally speaking, a formula $\varphi$ is a computable infinitary formula if it contains (finite or) infinite conjunctions or disjunctions, so long as they are taken over computably enumerable (c.e.) sets of computable infinitary formulas. More specifically, a computable infinitary $\Sigma_{0}^{0}$ or $\Pi_{0}^{0}$ formula is an open formula (i.e. a formula with only bounded quantifiers). Now, 
for any given computable ordinal $\alpha>0$, we define a computable infinitary $\Sigma_{\alpha}^{0}$ formula to be a (possibly) infinite disjunction of a c.e. set of formulas of the form $(\exists \bar{x}) \varphi_{i}(\bar{x})$, where $\varphi_{i}$ is a computable infinitary $\Pi_{\beta}^{0}$ formula, for some $\beta<\alpha$. The definition of a computable infinitary $\Pi_{\alpha}^{0}$ formula is similar, except we replace disjunction by conjunction, $\exists$ by $\forall$, and $\Pi_{\beta}^{0}$ by $\Sigma_{\beta}^{0}$. Also, the sets of computable infinitary $\Sigma_{\alpha}^{0}\left(A_{0}, A_{1}, \ldots, A_{k}\right), \Pi_{\alpha}^{0}\left(A_{0}, A_{1}, \ldots, A_{k}\right)$ formulas are defined similarly, except that we allow the sets $A_{0}, A_{1}, \ldots, A_{k}$ to appear as parameters in our formulas. $X \subset \omega$ is $\Sigma_{\alpha}^{0}$ (relative to $A_{0}, A_{1}, \ldots, A_{k}$ ) if it can be defined by a $\Sigma_{\alpha}^{0}$ formula (with parameters $A_{0}, A_{1}, \ldots, A_{k}$ ). A formula $\varphi$ is computable infinitary (relative to $\left.A_{0}, A_{1}, \ldots, A_{k}\right)$ if it is $\Sigma_{\alpha}^{0}\left(\Sigma_{\alpha}^{0}\left(A_{0}, A_{1}, \ldots, A_{k}\right)\right)$, for some computable ordinal $\alpha<\omega_{1}^{C K}$. For further information on computably infinitary formulas and their relation to the hyperarithmetic hierarchy, consult $\mathrm{AK}$.

\section{ABW AND $_{0} \mathrm{SL}_{0}$ ARE THEORIES OF HYPERARITHMETIC ANALYSIS}

The purpose of this section is the proof of the following theorem (i.e. Theorem 2.1). As we already said in the introduction, implications (1)-(3) below are stated (without proof) in [Fri75, page 239].

Theorem 2.1. The following implications hold over $\mathrm{RCA}_{0}+\mid \Sigma_{1}^{1}$.

(1) $\Sigma_{1}^{1}-\mathrm{AC}_{0} \rightarrow \mathrm{SL}_{0}$.

(2) $\Sigma_{1}^{1}-\mathrm{AC}_{0} \rightarrow \mathrm{ABW}_{0}$.

(3) $\mathrm{SL}_{0} \rightarrow \Sigma_{1}^{1}-\mathrm{AC}_{0}$ (and hence $\Sigma_{1}^{1}-\mathrm{AC}_{0} \leftrightarrow \mathrm{SL}_{0}$ ).

(4) $\mathrm{ABW}_{0} \rightarrow$ weak $-\Sigma_{1}^{1}-\mathrm{AC}_{0}$.

More precisely, we have that $\mathrm{SL}_{0} \leftrightarrow \Sigma_{1}^{1}-\mathrm{AC}_{0}$ over $\mathrm{RCA}$ (without $\mid \Sigma_{1}^{1}$ ).

Before we begin the proof of Theorem 2.1, we require the following lemma. Recall that every known theory of hyperarithmetic analysis that is not equal to either $\mathrm{SL}_{0}$ or $A B W_{0}$ implies $A C A_{0}$. The following lemma says that the same holds for $S_{0}$ and $\mathrm{ABW}_{0}$.

Lemma 2.2. Over $\mathrm{RCA}+\mathrm{A}_{0}+\Sigma_{1}^{1}$ we have that:

(1) $\mathrm{SL}_{0} \rightarrow \mathrm{ACA}_{0}$.

(2) $\mathrm{ABW}_{0} \rightarrow \mathrm{ACA}_{0}$.

Proof. We reason in $\mathrm{RCA}_{0}+\mathrm{I} \Sigma_{1}^{1}$.

To prove that a subsystem of second order arithmetic $S$ implies $A C A_{0}$, it suffices to show that $S$ implies the existence of the halting set $\emptyset^{\prime} \subseteq \mathbb{N}$. To show that $\mathrm{SL}_{0}$ implies the existence of the halting set $\emptyset^{\prime}$, let $A(X)$ be the arithmetic predicate which says that there is some $n \in \mathbb{N}$ such that $X\left\lceil(2 n+1)\right.$ is equal to $0^{n} 1 \emptyset^{\prime}\lceil n$, and $X=0^{n} 1 X\left\lceil n 0^{\infty}\right.$. Then, we have that every neighborhood of $0^{\infty}$ contains at least two solutions to $A(X)$, and therefore, by $\mathrm{SL}_{0}$, there is a sequence of reals, $\bigoplus_{n \in \mathbb{N}} X_{n}$, such that for all $n \in \mathbb{N}, A\left(X_{n}\right)$ holds. Via RCA 0 , we can assume without loss of generality that for every $k \in \mathbb{N}, X_{k}$ satisfies $X_{k}=0^{m} 1 \emptyset^{\prime}\lceil m$ for some $m>k$. Then it is clear that, for any given $n \in \mathbb{N}$, the first $n$ bits of $\emptyset^{\prime}$ can be computed (uniformly) from $X_{n}$ (so, via $\mathrm{RCA}_{0}$, we have that $\emptyset^{\prime} \subseteq \mathbb{N}$ exists).

To prove that $\mathrm{ABW}_{0}$ implies the existence of the halting set $\emptyset^{\prime}$, let $A(X)$ be the arithmetic predicate which says that $X$ is of the form $X=\sigma 0^{\infty}$, where $\sigma \in 2^{<\mathbb{N}}$, and $\sigma=\emptyset^{\prime} \uparrow|\sigma|$. Now, since $\emptyset^{\prime} \subseteq \mathbb{N}$ is an infinite/coinfinite set, it follows that $A(X)$ does not have finitely many solutions. Therefore, by $\mathrm{ABW}_{0}, A(X)$ must have an accumulation point. Now, by $I \Sigma_{1}^{1}$, for every $n \in \mathbb{N}$ there are at most $n$-many 
solutions of $A(X)$ such that $X\left\lceil n \neq \emptyset^{\prime} \uparrow n\right)$. Therefore, we have that $\emptyset^{\prime}$ is the unique accumulation point of $\{X: A(X)\}$, and by $\mathrm{ABW}_{0}, \emptyset^{\prime}$ exists.

Note that we only used the hypothesis $I \Sigma_{1}^{1}$ in the second paragraph to show that $A B W_{0}$ implies $A C A_{0}$. Therefore, (1) of Lemma 2.2 is valid over $R C A_{0}$.

Proof of Theorem 2.1. We reason in $\mathrm{RCA}_{0}+\mathrm{I}_{1}^{1}$. Note that, by Lemma 2.2 above, if $\mathrm{S} \in\left\{\Sigma_{1}^{1}-\mathrm{AC}_{0}, \mathrm{SL}_{0}, \mathrm{ABW}_{0}\right.$, weak $\left.-\Sigma_{1}^{1}-\mathrm{AC}_{0}\right\}$, then $\mathrm{S}$ implies $\mathrm{ACA}_{0}$, and therefore we will assume that $A C A_{0}$ holds throughout the proof of Theorem 2.1. We prove the four implications in order. Before we begin the proof of Theorem 2.1, we require the following elementary observations, definitions, and notation.

Now, let

$$
V=\left\{X \in 2^{\mathbb{N}}:\left(\exists^{\infty} n\right)[X(n)=1]\right\}
$$

we will refer to $V \subset 2^{\mathbb{N}}$ as the set of irrational numbers, and the complement of $V$ (in $2^{\mathbb{N}}$ ) as the set of rational numbers. Note that there is a natural computable homeomorphism $Z: V \rightarrow \mathbb{N}^{\mathbb{N}}$ such that $Z(X)(n), X \in V, n \in \mathbb{N}$, is equal to the number of 0 s between the $(n-1)^{\text {th }}$ and $n^{\text {th }} 1$ s appearing in $X \in V$. Note also that for every $X \in V, Z(X) \equiv_{T} X$.

To prove (1), first assume $\Sigma_{1}^{1}-\mathrm{AC}_{0}$. Now, let $A(X)$ be an arithmetic predicate with a single free set variable $X$. Furthermore, let $X_{0} \in 2^{\mathbb{N}}$ be such that for every open neighborhood $U \subseteq 2^{\mathbb{N}}$ of $X_{0}$, there exist $X_{1}, X_{2} \in U, X_{1} \neq X_{2}$, such that both $A\left(X_{1}\right)$ and $A\left(X_{2}\right)$ hold. We shall use $\Sigma_{1}^{1}-\mathrm{AC}_{0}$ to construct a set $Y \in 2^{\mathbb{N}}$ such that for every $k \in \mathbb{N}, A\left(Y^{[k]}\right)$ holds and $\lim _{k \rightarrow \infty} Y^{[k]}=X_{0}$, thus proving that $\Sigma_{1}^{1}-\mathrm{AC}_{0} \rightarrow \mathrm{SL}_{0}$.

Let $B(X, n), X \in 2^{\mathbb{N}}, n \in \mathbb{N}$, be the arithmetic predicate with a single free set variable $X$, and a single free number variable $n$, such that

$$
B(X, n) \equiv A(X) \wedge\left(X \left\lceiln=X_{0}\lceil n) .\right.\right.
$$

For any $X \in 2^{\mathbb{N}}$ and $n \in \mathbb{N}, B(X, n)$ says that $A(X)$ holds and $A\left\lceil n=X_{0}\lceil n\right.$. Now, by hypothesis (above), we know that for every $n \in \mathbb{N}$ there exists a $Y \in 2^{\mathbb{N}}$ such that $B(Y)$. Therefore, by $\Sigma_{1}^{1}-\mathrm{AC}_{0}$, there exists a set $Y \in 2^{\mathbb{N}}$ such that for every $k \in \mathbb{N}$ we have that $B\left(Y^{[k]}, k\right)$ holds. By definition of $B(X, n)$, this implies that for all $k \in \mathbb{N}$ we have that $Y^{[k]}\left\lceil k=X_{0}\left\lceil k\right.\right.$ (and so $\lim _{k \rightarrow \infty} Y^{[k]}=X_{0}$ ) and $A\left(Y^{[k]}\right.$ ) holds.

To prove (2), assume $\Sigma_{1}^{1}-\mathrm{AC}_{0}$ (recall that $\Sigma_{1}^{1}-\mathrm{AC}_{0}$ implies $A C \mathrm{~A}_{0}$ ), and let $A(X)$ be a bounded arithmetic predicate with a single free set variable $X$. We need to show that if for every $n \in \mathbb{N}, A$ has at least $n$-many solutions, then $A$ has an accumulation point $X_{0} \in 2^{\mathbb{N}}$ (note that $X_{0}$ need not satisfy $A$ ). Define an arithmetic predicate $B(X, n)$ with a single free set variable $X$ and a single free number variable $n$, such that

$$
B(X, n) \equiv\left(X=\bigoplus_{i=0}^{n} X^{i}\right) \wedge(\forall i \leq n)\left[A\left(X^{i}\right)\right] \wedge(\forall i, j \leq n)\left[i \neq j \rightarrow X^{i} \neq X^{j}\right] .
$$

For a fixed $n \in \mathbb{N}, B(X, n)$ says that $X \in 2^{\mathbb{N}}$ is the join of $n$-many distinct solutions to $A(X)$. We shall apply $\Sigma_{1}^{1}-\mathrm{AC}_{0}$ to the predicate $B(X)$ in order to prove that $\mathrm{ABW}_{0}$ holds for the predicate $A(X)$. To do this, we first need to prove that, for every $n \in \mathbb{N}$, there exists $X_{n} \in 2^{\mathbb{N}}$ such that $B\left(X_{n}, n\right)$ holds. This follows from $\mid \Sigma_{1}^{1}$ 
and our assumption that for all $n \in \mathbb{N}, A(X)$ has at least $n$-many solutions. Hence, by $\Sigma_{1}^{1}-\mathrm{AC}_{0}$, we have that there exists a set

$$
X=\bigoplus_{n \in \mathbb{N}} X_{n}=\bigoplus_{n \in \mathbb{N}} \bigoplus_{i=0}^{n} X_{n}^{i}
$$

such that $B\left(X_{n}, n\right)$ holds for every $n \in \mathbb{N}$. Note that, by our definition of $B(X, n)$, $X_{n}^{i}$ satisfies $A\left(X_{n}^{i}\right)$ for all $n \in \mathbb{N}, 0 \leq i \leq n$. Moreover, if for every $n \in \mathbb{N}$ we define

$$
Y_{n}=\bigoplus_{\substack{0 \leq m \leq n \\ 0 \leq i \leq m}} X_{m}^{i},
$$

then $Y_{n}$ contains at least $n$-many distinct columns because $X_{n}^{i}, 0 \leq i \leq n$, are columns of $Y_{n}$. Using this fact, and arithmetic comprehension (i.e. $\mathrm{ACA}_{0}$ ) relative to $X$, we can construct a set $Y \in 2^{\mathbb{N}}$ such that $Y$ is of the form

$$
Y=\bigoplus_{n \in \mathbb{N}} Y_{n}
$$

and for every $n \in \mathbb{N}$ we have that $A\left(Y_{n}\right)$ holds and $Y_{0}, Y_{1}, \ldots, Y_{n}, \ldots \in 2^{\mathbb{N}}$ are mutually distinct.

Now, using $\mathrm{ACA}_{0}$ relative to $Y$, we may construct a tree $T \subseteq 2^{<\mathbb{N}}$ via the following definition:

$$
T=\left\{\sigma \in 2^{<\mathbb{N}}:\left(\exists^{\infty} n \in \mathbb{N}\right)\left[\sigma=Y_{n} \uparrow|\sigma|\right]\right\} .
$$

$T \subseteq 2^{<\mathbb{N}}$ consists of all nodes $\sigma \in 2^{<\mathbb{N}}$ that are initial segments of $Y_{n}$ for infinitely many $n \in \mathbb{N}$. We now wish to show that $T$ contains infinitely many nodes. Then, since we are assuming $A_{C A}$, we can conclude that there exists an infinite path $Z \in 2^{\mathbb{N}}$ through $T$. Lastly, we will show that $Z$ is in fact an accumulation point of the set $\left\{X \in 2^{\mathbb{N}}: A(X)\right\}$. Let $n \in \mathbb{N}$ be given. We shall show that $T$ contains a node of length $n$. Note that there are $2^{n}$-many binary strings of length $n$, and by $\mathrm{ACA}_{0} 5$ it follows that for some $\sigma \in 2^{<\mathbb{N}},|\sigma|=n$, there exist infinitely many $m \in \mathbb{N}$ such that $\sigma=Y_{m}\lceil n$. By definition of $T$, we have that $\sigma \in T$. Hence, $T$ is infinite. Now, using $\mathrm{ACA}_{0}$ with the parameter $T$, we can construct an infinite path $Z \in 2^{\mathbb{N}}$ through $T$. We now claim that $Z$ is an accumulation point of the set $\left\{X \in 2^{\mathbb{N}}: A(X)\right\}$. Let $n \in \mathbb{N}$ be given. We must construct a real $X \in 2^{\mathbb{N}}$ such that $Z\lceil n=X\lceil n, Z \neq X$, and $A(X)$ holds. Since $Z\lceil n \in T$, (by definition of $T$ ) we know that there are infinitely many $m \in \mathbb{N}$ such that $Y_{m}\left\lceil n=Z\left\lceil n\right.\right.$ and $A\left(Y_{m}\right)$. Now, since $Y_{0}, Y_{1}, Y_{2}, \ldots$ are mutually distinct sets, we can find some $Y_{m}, m \in \mathbb{N}$, such that $Y_{m} \neq Z, Y_{m}\left\lceil n=Z\left\lceil n\right.\right.$, and $A\left(Y_{m}\right)$ holds. This proves that $Z \in 2^{\mathbb{N}}$ is indeed an accumulation point for the set $\left\{X \in 2^{\mathbb{N}}: A(X)\right\}$.

To prove (3), assume that $\mathrm{SL}_{0}$ holds, and suppose that $A(X, n)$ is an arithmetic predicate with one free set variable $X$ and one free number variable $n$ such that for every $m \in \mathbb{N}$ there exists $X \in 2^{\mathbb{N}}$ such that $A(X, m)$ holds. We need to construct a set $Y=\bigoplus_{n \in \mathbb{N}} Y_{n}$ such that, for every $n \in \mathbb{N}$, we have $A\left(Y_{n}, n\right)$. Without loss of

\footnotetext{
${ }^{5} A C A_{0}$ implies $B \Sigma_{2}$, which is equivalent to the infinite pigeonhole principle. The infinite pigeonhole principle states that if $X \subseteq \mathbb{N}$ is infinite and we have that $X=\bigcup_{i=0}^{n} X_{i}$, then there is an $i \in\{0,1,2, \ldots, n\}$ such that $X_{i}$ is infinite.
} 
generality, assume that $A(X, 0)$ has a solution of the form $0 X, X \in 2^{\mathbb{N}}$, and define an arithmetic predicate $B(X), X \in 2^{\mathbb{N}}$, as follows:

$$
\begin{array}{r}
B(X) \equiv(\exists n \in \mathbb{N})(\forall m \in \mathbb{N})\left[\left(m<n \rightarrow X^{[m]}=0^{\infty}\right) \wedge\left(m>n \rightarrow X^{[m]}=10^{\infty}\right) \wedge\right. \\
\left.\left(m=n \rightarrow\left(X^{[n]}=\bigoplus_{i=0}^{n} X_{i}^{n}\right) \wedge\left(X^{[n]}(0)=0\right) \wedge(\forall i \leq n)\left[A\left(X_{i}^{n}, i\right)\right]\right)\right] .
\end{array}
$$

$B(X)$ says that there exists a number $n \in \mathbb{N}$ such that every row of $X, X^{[m]}, m \in \mathbb{N}$, is equal to $0^{\infty}$ if $m<n$ or $10^{\infty}$ if $m>n$, except for possibly the single row, $X^{[n]}$, which is the join of the sets $X_{0}^{n}, X_{1}^{n}, \ldots, X_{n}^{n}$ such that for all $0 \leq i \leq n, X_{i}^{n}$ satisfies $A\left(X_{i}^{n}, i\right)$. If $A(X, 0)$ has no solution of the form $0 X, X \in 2^{\mathbb{N}}$, then replace $X^{[n]}(0)=0$ in the definition of $B(X)$ with $X^{[n]}(0)=1$. The rest of the proof would change only slightly in this case. We assume that after having read the rest of our proof below, the reader could supply the proof of Theorem 2.1 (3) in the case where $A(X, 0)$ has no solution of the form $0 X, X \in 2^{\mathbb{N}}$ (and therefore we will neglect to consider this case in our proof below).

Let $n \in \mathbb{N}$ be given, and (by $\mid \Sigma_{1}^{1}$ ) let $X_{0}^{n}, X_{1}^{n}, \ldots, X_{n}^{n} \in 2^{\mathbb{N}}$ be such that $A\left(X_{i}^{n}, i\right)$ holds for all $0 \leq i \leq n$. By definition of $B(X)$, the set $X_{n} \in 2^{\mathbb{N}}, n \in \mathbb{N}$, defined by $X_{n}^{[k]}=0^{\infty}$ if $k<n, X_{n}^{[k]}=10^{\infty}$ if $k>n$, and $X_{n}^{[n]}=\bigoplus_{i=0}^{n} X_{i}^{n}$, satisfies $B\left(X_{n}\right)$. Since, by assumption, there exists a number $m \in \mathbb{N}$ such that $A(X, m) \rightarrow X \neq 0^{\infty}$, then it follows that every neighborhood of $0^{\infty}$ contains at least two solutions to $B(X)$. Therefore, by $\mathrm{SL}_{0}, 0^{\infty}$ is the limit of some sequence of solutions to $B(X)$. In other words, there exists $Y=\bigoplus_{n \in \mathbb{N}} Y_{n} \in 2^{\mathbb{N}}$ such that $\lim _{n \rightarrow \infty} Y_{n}=0^{\infty}$ and for all $n \in \mathbb{N}, B\left(Y_{n}\right)$ holds.

Notice that $Y$ must (uniformly) code solutions to $A(X, n)$, for all $n \in \mathbb{N}$. To see this, for all $n \in \mathbb{N}$ (via $\mathrm{ACA}_{0}$ ) let $f(n) \in \mathbb{N}$ be the largest natural number such that for all $m \leq f(n)$ we have that $Y_{n}^{[m]}(0)=0$. By our construction of $B(X)$ above, it follows that, for every $n \in \mathbb{N}, f(n)$ exists and $X=Y_{n}^{[f(n)]}$ satisfies $A\left(X^{[k]}, k\right), 0 \leq k \leq f(n)$. In other words, $Y_{n}^{[f(n)]}$ codes solutions to $A(X, k)$ for $k=0,1, \ldots, f(n)$. Also, note that since $\lim _{n \rightarrow \infty} Y_{n}=0^{\infty}$, it follows that $\lim _{n \rightarrow \infty} f(n)=\infty$.

Now, from $Y=\bigoplus_{n \in \mathbb{N}} Y_{n}$ and $f(n)$, we shall uniformly compute a set $X=$ $\bigoplus_{n \in \mathbb{N}} X_{n}$ such that for every $n \in \mathbb{N}, A\left(X_{n}, n\right)$ holds. To compute $X^{[n]}$ (using the fact that $\lim _{n} f(n)=\infty$ ) find the smallest argument $m \in \mathbb{N}$ such that $f(m)>n$, and set $X^{[n]}$ equal to $\left(Y_{m}^{[f(m)]}\right)^{[n]}$ (i.e. the $n^{\text {th }}$ column of the $f(m)^{\text {th }}$ column of $Y_{m}$ ). By our construction of $B(X)$ above, we have that $A\left(X^{[n]}, n\right)$ holds, as required.

To prove implication (4), assume $\mathrm{ABW}_{0}$, and suppose that $A(X, n)$ is an arithmetic predicate with a single free set variable $X$ and a single free number variable $n$ such that for every $n \in \mathbb{N}$ there exists a unique $X \in \mathbb{N}^{\mathbb{N}}$ such that $A(X, n)$ holds. Recall our definitions of $V \subseteq 2^{\mathbb{N}}$ and $Z: V \rightarrow \mathbb{N}^{\mathbb{N}}$ given at the beginning of this proof. Via $\mathrm{ABW}_{0}$, we shall construct the set $Y \in 2^{\mathbb{N}}$ such that $Y=\bigoplus_{n \in \mathbb{N}} X_{n}$ and $A\left(Z\left(X_{n}\right), n\right)$ (it is not difficult to check that, over $\mathrm{RCA}_{0}$, constructing such a $Y \in 2^{\mathbb{N}}$ is equivalent to proving that weak choice holds for $\left.A(X, n)\right)$. To construct $Y \in 2^{\mathbb{N}}$, consider the bounded arithmetic predicate $B(X)$ defined by

$$
B(X) \equiv\left(\exists n_{0} \in \mathbb{N}\right)\left[\left(\forall n \leq n_{0}\right)\left[A\left(Z\left(X^{[n]}\right), n\right)\right] \wedge\left(\forall n>n_{0}\right)\left[X^{[n]}=0^{\infty}\right]\right] .
$$


$B(X)$ says that there exists a number $n_{0} \in \mathbb{N}$ such that if $n \leq n_{0}$, then $X^{[n]} \in 2^{\mathbb{N}}$ satisfies $A\left(Z\left(X^{[n]}\right), n\right)$, and if $n>n_{0}$, then $X^{[n]}=0^{\infty}$. By $\mid \Sigma_{1}^{1}$, and the fact that $0^{\infty} \notin V$, it follows that for all $k \in \mathbb{N}, B(X)$ has at least $k$-many solutions.

Now, we can apply $\mathrm{ABW}_{0}$ to conclude that there exists $Y \in 2^{\mathbb{N}}$ such that $Y$ is an accumulation point for the set $\left\{X \in 2^{\mathbb{N}}: B(X)\right\}$. Note that, for any given number $k \in \mathbb{N}, Y$ is also an accumulation point for the class $\left\{X \in 2^{\mathbb{N}}: B(X)\right\} \cap\{X \in$ $\left.V:(\forall i \leq k)\left[A\left(Z\left(X^{[k]}\right), k\right)\right]\right\}$, since this class differs from $\left\{X \in 2^{\mathbb{N}}: B(X)\right\}$ by at most $k$-many elements. We now show that $Y=\bigoplus_{n \in \mathbb{N}} X_{n}$, where $X_{n}, n \in \mathbb{N}$, is such that $A\left(Z\left(X_{n}\right), n\right)$ holds. It suffices to show that, for every $n \in \mathbb{N}$ and $k \leq n$, we have that $Y^{[k]} \mid n=X\left\lceil n\right.$, where $X \in 2^{\mathbb{N}}$ is the unique solution to $A(Z(X), k)$. Suppose, for a contradiction, that this were not the case. In other words, suppose that for some $n \in \mathbb{N}$ there is some $k \in \mathbb{N}, k \leq n$, such that $Y^{[k]} \mid n \neq X_{0}\left\lceil n, X_{0} \in V\right.$, and $A\left(Z\left(X_{0}\right), k\right)$ holds. Then, since $Y \in 2^{\mathbb{N}}$ is an accumulation point for the class $\left\{X \in 2^{\mathbb{N}}: B(X)\right\} \cap\left\{X \in V:(\forall i \leq k)\left[A\left(Z\left(X^{[i]}\right), i\right)\right]\right\}$, there exists $W \in\left\{X \in 2^{\mathbb{N}}\right.$ : $B(X)\} \cap\left\{X \in V:(\forall i \leq k)\left[A\left(Z\left(X^{[i]}\right), i\right)\right]\right\}$ such that $Y^{[k]}\left\lceil n=W^{[k]}\left\lceil n \neq X_{0}\lceil n\right.\right.$ and $B(W)$ holds. Now, by definition of $X_{0}, W \in V$, we have that $A\left(Z\left(X_{0}\right), k\right)$ holds, $A\left(Z\left(W^{[k]}\right), k\right)$ holds, but $Z\left(X_{0}\right) \neq Z\left(W^{[k]}\right)$, and therefore we have contradicted the fact that for every $m \in \mathbb{N}$ there is a unique set $X \in \mathbb{N}^{\mathbb{N}}$ such that $A(X, m)$ holds. We now conclude that $Y \in 2^{\mathbb{N}}, Y=\bigoplus_{n \in \mathbb{N}} X_{n}, A\left(Z\left(X_{n}\right), n\right)$, as required.

At this point we wish to note that we did not use our hypothesis of $I \Sigma_{1}^{1}$ in the proofs of (1) and (3) above; therefore, we have that $\mathrm{SL}_{0} \leftrightarrow \Sigma_{1}^{1}-\mathrm{AC}_{0}$ holds over $\mathrm{RCA}_{0}$.

Corollary 2.3. $\mathrm{G}_{\delta \sigma} \mathrm{ABW}_{0}$ is a theorem of hyperarithmetic analysis.

Proof. In [Mon06, page 113] it is shown that the restriction of weak $-\Sigma_{1}^{1}-\mathrm{AC}_{0}$ to two quantifier arithmetic predicates of the form $\forall \exists \cdots$ is a theorem of hyperarithmetic analysis (since it implies CDG - CA, which is equivalent to $\mathrm{L}_{\omega_{1}, \omega} \mathrm{CA}_{0}$ ). If we take the predicate $A(X, n)$ of Theorem 2.1 (4) to be of this form, then it follows that the predicate $B(X)$ is of the form $\exists \forall \exists \cdots$ (i.e. $B$ is $\Sigma_{3}^{0}$ relative to some parameter), which corresponds to a $G_{\delta \sigma}$ set of real numbers. Therefore, to prove the restriction of weak $-\Sigma_{1}^{1}-A C_{0}$ to two quantifier arithmetic predicates of the form $\exists \forall \exists \cdots$ it suffices to assume $\mathrm{G}_{\delta \sigma} \mathrm{ABW}_{0}$. It follows that $\mathrm{G}_{\delta \sigma} \mathrm{ABW}_{0}$ is a theorem of hyperarithmetic analysis.

Next, we shed light on the reverse mathematical strengths of $\mathrm{OABW}, \mathrm{CABW}_{0}$, $\mathrm{F}_{\sigma} \mathrm{ABW}_{0}, \mathrm{G}_{\delta} \mathrm{ABW}_{0}$, and $\mathrm{F}_{\sigma \delta} \mathrm{ABW}_{0}$.

Theorem 2.4. The following hold over $\mathrm{RCA}_{0}$.

(1) $\mathrm{OABW}_{0}$ is equivalent to $\mathrm{RCA}_{0}$.

(2) $\mathrm{CABW}_{0}$, and $\mathrm{F}_{\sigma} \mathrm{ABW}_{0}$ are equivalent to $\mathrm{ACA}_{0}$.

(3) $\mathrm{G}_{\delta} \mathrm{ABW}_{0}$ and $\mathrm{F}_{\sigma \delta} \mathrm{ABW}_{0}$ imply $\mathrm{ACA}_{0}$.

Proof. Note that $\mathrm{F}_{\sigma} \mathrm{ABW}_{0}$ implies $\mathrm{CABW}_{0}$, and that $\mathrm{F}_{\sigma \delta} \mathrm{ABW}_{0}$ implies $\mathrm{G}_{\delta} \mathrm{ABW}_{0}$ (since every open set is $F_{\sigma}$, even in $\mathrm{RCA}_{0}$ ).

To prove (1) it suffices to prove $\mathrm{OABW}_{0}$ via $\mathrm{RCA}_{0}$. Let $U \subseteq 2^{\mathbb{N}}$ be a bounded open set of reals (recall that in $\mathrm{RCA}_{0}$, bounded open sets of reals are represented by a prefix-free sequence of finite binary strings), and let $\sigma \in 2^{<\mathbb{N}}$ be such that the open set generated by $\sigma,[\sigma] \subseteq 2^{\mathbb{N}}$, is contained in $U$. It follows that $\sigma 0^{\infty}$ exists 
(via $\mathrm{RCA}_{0}$ ) and is the accumulation point of the sequence $f_{n}=\sigma 0^{n} 1^{\infty} \in U, n \in \mathbb{N}$. Note that the sequence $\left\{f_{n}\right\}_{n \in \mathbb{N}}$ exists via $\operatorname{RCA}_{0}$.

We now sketch the proof of (2) and leave it to the reader to fill in the details. First, we show that $\mathrm{ACA}_{0}$ implies $\mathrm{F}_{\sigma} \mathrm{ABW}_{0}$. To see why, let $C_{n} \subseteq 2^{<\mathbb{N}}, n \in \mathbb{N}$, be a sequence of bounded (effectively) closed sets (relative to some parameter). We aim to show that $\bigcup_{n \in \mathbb{N}} C_{n} \subseteq 2^{\mathbb{N}}$ is either finite or has an accumulation point. If $\bigcup_{n \in \mathbb{N}} C_{n} \subseteq 2^{\mathbb{N}}$ is not finite, then via $\mathrm{ACA}_{0}$ we can construct a sequence of mutually distinct reals, $\left\{f_{j}\right\}_{j \in \mathbb{N}}, f_{j} \in 2^{\mathbb{N}}$, such that for all $j \in \mathbb{N}$ there exists $n \in \mathbb{N}$ such that $f_{j} \in C_{n}$. Then, we may apply the standard Bolzano-Weierstrass theorem Sim, Theorem III.2.2] (via $\mathbf{A C A}_{0}$ ) to the bounded sequence of reals $\left\{f_{j}\right\}_{j \in \mathbb{N}}$ to obtain a convergent subsequence. Finally, we may use $\mathrm{ACA}_{0}$ (again) to take the limit of the subsequence, thus obtaining an accumulation point for the convergent subsequence (which is also an accumulation point for the sequence $\left\{f_{j}\right\}_{j \in \mathbb{N}}$ and also the $F_{\sigma}$ set $\bigcup_{n \in \mathbb{N}} C_{n} \subseteq 2^{\mathbb{N}}$ ). To complete the proof of (2) it suffices to show that $\mathrm{CABW}_{0}$ implies $A C A_{0}$. To prove this, simply construct a $\Pi_{1}^{0}$-class with a unique rank one (i.e. nonisolated) point that computes the halting set. The construction is valid in $\mathrm{RCA}_{0}$ (here we are using the fact that RCA $\mathrm{A}_{0}$ includes induction for $\Sigma_{1}^{0}$ formulas).

Note that (3) follows from (2) (i.e. (3) follows from the fact that $\mathrm{CABW}_{0}$ implies $\mathrm{ACA}_{0}$ since every closed set of reals is $G_{\delta}$ and every $G_{\delta}$ set of reals is $\left.F_{\sigma \delta}\right)$.

In the next two sections we will show that $\mathrm{G}_{\delta} \mathrm{ABW}_{0}, \mathrm{~F}_{\sigma \delta} \mathrm{ABW}_{0}$, and $\mathrm{G}_{\delta \sigma} \mathrm{ABW}_{0}$

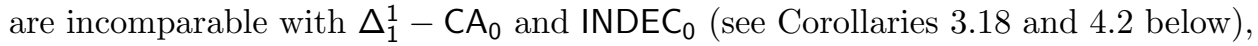
and that $\mathrm{G}_{\delta} \mathrm{ABW}_{0}$ and $\mathrm{F}_{\sigma \delta} \mathrm{ABW}_{0}$ are strictly stronger than $\mathrm{ACA}_{0}$ (see Corollary 3.19 below). We will obtain all of these results as a corollary to our proof that $\mathrm{ABW}_{0}$ is incomparable with $\Delta_{1}^{1}-\mathrm{CA}_{0}$ and INDEC 0 .

\section{3. $\Delta_{1}^{1}-\mathrm{CA}_{0}$ DOES NOT IMPLy $\mathrm{ABW}_{0}$}

In this section we prove the following theorem.

Theorem 3.1. There is an $\omega$-model of $\Delta_{1}^{1}-\mathrm{CA}_{0}$ that is not a model of $\mathrm{ABW}_{0}$. Hence, $\Delta_{1}^{1}-\mathrm{CA}_{0}$ does not imply $\mathrm{ABW}_{0}$.

In [Ste78, Steel constructs an $\omega$-model $M_{\infty} \cap \mathcal{P}(\omega)$ that satisfies $\Delta_{1}^{1}-\mathrm{CA}_{0}$, but not $\Sigma_{1}^{1}-A C_{0}$. In Mon, Montalbán shows that Steel's model also satisfies $\Pi_{1}^{1}-\mathrm{SEP}_{0}$. We shall provide a subtle modification of Steel's construction that produces a model $M_{\infty}$ satisfying $\Delta_{1}^{1}-\mathrm{CA}_{0}+\neg \mathrm{ABW}_{0}$. The rest of this section closely follows Montalbán's treatment of the matter in Section 2 of [Mon].

3.1. Constructing $M_{\infty}$. To construct $M_{\infty}$, we first construct a generic object

$$
G=\left\langle T^{G},\left\{\alpha_{i}^{G}: i \in \omega\right\}, h^{G}\right\rangle,
$$

where $T^{G} \subseteq \omega^{<\omega}$ is a tree, $\left\{\alpha_{i}: i \in \omega\right\}$ is a set of paths through $T^{G}$, and $h^{G}$ : $T^{G} \rightarrow \omega_{1}^{C K} \cup\{\infty\}$ is the well-founded rank function for $T^{G}$; i.e. $h^{G}(s)=|s|_{T^{G}}$. The function $h^{G}$ ensures that $\left[T^{G}\right] \cap M_{\infty}=\left\{\alpha_{i}: i \in \omega\right\}$, and helps to prove certain properties of the forcing notion.

Throughout this section $F$ will denote a finite subset of $\omega$, i.e. $F \subset_{f} \omega$. For all such $F$, we let $M_{F}$ be the class of sets that can be defined by a computable infinitary formula relative to the parameters $T^{G}$ and $\alpha_{i}, i \in F$. In other words,

$$
M_{F}=\left\{X \subseteq \omega:\left(\exists \mu<\omega_{1}^{C K}\right)\left[X \in \Sigma_{\mu}^{0}\left(T^{G}, \alpha_{i}^{G}: i \in F\right)\right]\right\} .
$$


This can also be expressed as follows:

$$
M_{F}=\mathcal{P}(\omega) \cap L_{\omega_{1}^{C K}}\left(\left\{T^{G}\right\} \cup\left\{\alpha_{i}: i \in F\right\}\right),
$$

where $L_{\omega_{1}^{C K}}\left(\left\{T^{G}\right\} \cup\left\{\alpha_{i}: i \in F\right\}\right)$ is the class of Gödel constructible sets up to level $\omega_{1}^{C K}$, starting from $\left\{T^{G}\right\} \cup\left\{\alpha_{i}^{G}: i \in F\right\}$ [Ste78, page 57]. Lemma 3.8 says that, for every $F \subset_{f} \omega$, we have that $M_{F}=\operatorname{HYP}\left(T \oplus \bigoplus_{i \in F} \alpha_{i}\right)$. It follows that, for every $F \subset_{f} \omega, M_{F}$ is closed under hyperarithmetic reductions.

We now define our desired model $M_{\infty}$ as follows:

$$
M_{\infty}=\bigcup_{F \subset_{f} \omega} M_{F} .
$$

As in [Ste78, Mon, we will show that for every $F \subset_{f} \omega$, the set of paths through $T^{G}$ in $M_{F}$ is equal to $\left\{\alpha_{i}: i \in F\right\}$. It follows that the set of paths through $T^{G}$ in $M_{\infty}$ is $\left\{\alpha_{i}: i \in \omega\right\}$ (Lemma 3.6), and from this fact we shall be able to deduce that $M_{\infty} \not \models \mathrm{ABW}_{0}$ (Corollary 3.7).

For any given $\mu<\omega_{1}^{C K}$ and $F \subset_{f} \omega$, we define $M_{\mu, F}, M_{\mu, \infty}, H_{F, \mu}$, and $S_{\mu, F, e}$ exactly as in Section 2 of [Mon. $M_{\mu, F}$ is the class of $\Sigma_{\nu}^{0}$-definable sets in the parameters $T$ and $\alpha_{i}, i \in F$, where $\nu$ ranges over all ordinals less than $\mu . M_{\mu, \infty}=$ $\bigcup_{F \subset_{f} \omega} M_{\mu, F} . H_{F, 1}$ is defined as the join of $T^{G}, \alpha_{i}, i \in F ; S_{\mu, F, e}$ is the $e^{\text {th }}$ c.e. set relative to $H_{F, \mu}$; and for $\mu>1, H_{F, \mu}$ is the join of $S_{\nu, F, e}$ such that $\nu<\mu, e \in \omega$. By our definitions above, and some well-known facts about infinitary formulas and definability, it follows that the sets $S_{\mu, F, e}$ belong to $\Sigma_{\mu}^{0}\left(T^{G}, \alpha_{i}: i \in F\right)$ and that $M_{\mu, F}=\left\{S_{\nu, F, e}: e \in \omega, \nu<\mu\right\}$.

3.2. Our forcing conditions. Our forcing conditions are motivated by those of Steel Ste78 and Montalbán Mon. One major difference, however, is the introduction of a new tagging function $g: T_{\infty}^{p} \rightarrow\{0,1\}$ that tags the set of nodes $T_{\infty}^{p}=\left\{\sigma \in T^{p}: h^{p}(\sigma)=\infty\right\}$ (see below for more details). The function $g$ ensures that we do not add too many paths to the generic tree $T^{G}=\bigcup_{p \in G} T^{p}$, which allows us to conclude that $\left[T^{G}\right]$ has no accumulation points in $M_{\infty}$, and therefore $M_{\infty}$ does not satisfy $\mathrm{ABW}_{0}$ (see Lemma 3.6 and Corollary 3.7).

Our forcing conditions are quadruplets $\left\langle T^{p}, f^{p}, h^{p}, g^{p}\right\rangle$ such that

(1) $T^{p} \subset \omega^{<\omega}$ is a finite nonempty tree.

(2) $f^{p}: \omega \rightarrow T^{p}$ is such that $\operatorname{dom}\left(f^{p}\right) \subset_{f} \omega$.

(3) $h^{p}: T^{p} \rightarrow \omega_{1}^{C K} \cup\{\infty\}$ so that

(a) $\left(\forall \sigma, \tau \in T^{p}\right)\left[\sigma \subset \tau \Rightarrow h^{p}(\sigma)>h^{p}(\tau)\right]$.

(b) $\left(\forall \sigma \in T^{p}\right)\left[\left((\exists i \in \omega) \sigma \subseteq f^{p}(i)\right) \Rightarrow h^{p}(\sigma)=\infty\right]$.

(c) $h^{p}(\emptyset)=\infty$.

(4) $g^{p}: T_{\infty}^{p} \rightarrow\{0,1\}$, where $T_{\infty}^{p}=\left\{\sigma \in T^{p}: h^{p}(\sigma)=\infty\right\}$, is such that if $T^{p}=\{\emptyset\}$, then $g^{p}(\emptyset)=1$.

By fiat, $\infty>\infty$ and $\infty>\omega_{1}^{C K}$. From now on, let $P$ denote the set of our forcing conditions.

For $p, q \in P$, we define $p \leq q$ if and only if

(5) $T^{q} \subseteq T^{p}$.

(6) (a) $\operatorname{dom}\left(f^{q}\right) \subseteq \operatorname{dom}\left(f^{p}\right)$.

(b) $\left(\forall i \in \operatorname{dom}\left(f^{q}\right)\right)\left[f^{q}(i) \subseteq f^{p}(i)\right]$.

(c) $\left(\forall i \in \operatorname{dom}\left(f^{q}\right)\right)\left(\nexists \sigma \in T^{q}\right)\left[f^{q}(i) \subset \sigma \subseteq f^{p}(i)\right]$.

(7) $h^{q}=h^{p} \uparrow T^{q}$. 
(8) (a) If $g^{q}(\sigma)=1$, but $g^{p}(\sigma)=0$, then there exists some $\tau \supset \sigma, \tau \in$ $T^{p}$, such that $\sigma \in T^{q}$ is the longest initial segment of $\tau$ on $T^{q}$, and $g^{p}(\tau)=1$.

(b) For all $\sigma \in T^{p} \backslash T^{q}$ such that $g^{p}(\sigma)=1$, there exists $\tau_{\sigma} \in T^{q}$, $\tau_{\sigma} \subset \sigma$ such that $g^{q}\left(\tau_{\sigma}\right)=1$ and $g^{p}\left(\tau_{\sigma}\right)=0$.

(9) For every $i \in \operatorname{dom}\left(f^{p}\right) \backslash \operatorname{dom}\left(f^{q}\right)$, if $\sigma_{i} \in T^{q}$ is the longest initial segment of $f^{p}(i)$ on $T^{q}$, then we have that $g^{q}\left(\sigma_{i}\right)=1$.

For any given $p \in P, T^{p}, h^{p}$, and $f^{p}$ play the same role here as they did in Mon, Ste78. The most significant difference between our conditions and those of [Mon, Ste78] is the introduction of our function $g^{p}: T_{\infty}^{p} \rightarrow\{0,1\}$ that tags the nodes of $T_{\infty}^{p}=\left\{\sigma \in T^{p}: h^{p}(\sigma)=\infty\right\}$. More precisely, $g^{p}$ acts as a lock on $\sigma \in T_{\infty}^{p}$. The lock is open when $g^{p}(\sigma)=1$, and the lock is closed when $g^{p}(\sigma)=0$. Condition (9) says that if $p \leq q$ wishes to add new paths to our model by extending the domain of $f^{q} \subset f^{p}$, then the longest initial segment of all such paths in $T^{q}$ must be unlocked. The primary goal of the lock $g^{p}(\sigma)$ is to restrict the creation of new paths, so that we can prove that $[T] \subset \omega^{\omega}$ has no accumulation points in $M_{\infty}$,

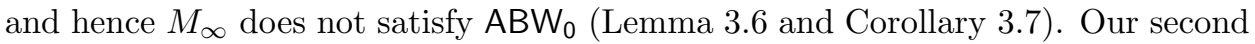
requirement on $g$ is given in condition (8) above. Roughly speaking, condition (8a) above says that whenever we lock a node $\sigma \in T^{q}$, there must be some node $\tau \supset \sigma, \tau \in T^{p} \backslash T^{q}$, that is unlocked. This property will be used to prove that $M_{\infty}$ satisfies $\Delta_{1}^{1}-\mathrm{CA}_{0}$ (Lemma 3.13). Condition (8b) is a converse to (8a) and says that in order to unlock a node $\tau \in T^{p}$, we must lock some initial segment of $\tau$. We will use condition (8b) in the proof of Corollary 3.7 below, which says that $A B W_{0}$ does not hold in our model $M_{\infty}$.

Let $\boldsymbol{P}=\langle P, \leq\rangle$, and let $\boldsymbol{G} \subset \boldsymbol{P}$ be a sufficiently $\boldsymbol{P}$-generic filter. More specifically, let $\boldsymbol{G}$ be generic enough to force every $\Sigma$-over- $\mathcal{L}_{F}$ formula, which we will define later in this section. Define $T^{G}=\bigcup_{p \in G} T^{p}, \alpha_{i}^{G}=\bigcup_{p \in G} f^{p}(i), i \in \omega$, and $h^{G}=\bigcup_{p \in G} h^{p}$. By definition of $M_{\infty}=\bigcup_{F \subset_{f} \omega} M_{F}$, we will show that for all $i \in \omega$, we have that $\left[T^{G}\right] \cap M_{\infty}=\left\{\alpha_{i}^{G}: i \in \omega\right\}$. From this it will follow that $M_{\infty}$ does not satisfy $A B W_{0}$.

3.3. The forcing language. The forcing language $\mathcal{L}_{\infty}$, as well as the languages $\mathcal{L}_{F}, F \subset_{f} \omega$, are identical to those defined by Montalbán in Section 2.3 of [Mon]. For a complete description of Montalbán's languages, we refer the reader to [Mon]. Here we will give a brief overview of our languages, which we denote by $\mathcal{L}_{\infty}$ and $\mathcal{L}_{F}, F \subset_{f} \omega$.

The languages $\mathcal{L}_{F}, F \subset_{f} \omega$, consist of the symbols $\in=,+, \times, \leq$; constants for natural numbers; number variables; unranked set variables $X_{H}, Y_{H}, \ldots, H \subseteq F$; ranked set variables $X_{H}^{\nu}, Y_{H}^{\nu}, \ldots, H \subseteq F$; the usual logical connectives; the usual quantifiers for both number and set variables; the symbols $\boldsymbol{T}, \boldsymbol{\alpha}_{i}, \boldsymbol{S}_{\nu, F, e}, \boldsymbol{H}_{\nu, F}: i \in$ $F \subset_{f} \omega, e \in \omega, \nu<\omega_{1}^{C K}$; and the elements of the sets of constants $C_{\lambda}^{F}, \lambda<\omega_{1}^{C K}$, that name elements of $M_{F}$.

The symbols of $\mathcal{L}_{\infty}$ include those in $\bigcup_{F \subset_{f} \omega} \mathcal{L}_{F}$, but also include both ranked and unranked set variables of the form $X^{\nu}$ and $X$, respectively. A variable $X \in \mathcal{L}_{\infty}$ is $F$-restricted if it is subscripted $H$ for some $H \subseteq F$. A formula of $\mathcal{L}_{\infty}$ is $F$-restricted if and only if all of its bounded variables are $F$-restricted.

The semantics of the languages $\mathcal{L}_{\infty}, \mathcal{L}_{F}, F \subset_{f} \omega$ are straightforward. Simply remember that $\boldsymbol{T}$ denotes $T^{G} \subseteq \omega^{<\omega} ; \boldsymbol{\alpha}_{i}$ denotes $\alpha_{i}^{G} \in \omega^{\omega} ; \boldsymbol{S}_{\nu, F, e}$ denotes $S_{\nu, F, e}$; 
$\boldsymbol{H}_{\nu, F}$ denotes $H_{\nu, F} ; X_{F}^{\nu}$ ranges over $M_{\nu, F} ; X^{\nu}$ ranges over $M_{\nu, \infty} ; X_{F}$ ranges over $M_{F}$; and $X$ ranges over $M_{\infty}$.

We say that a formula of $\mathcal{L}_{\infty}$ is ranked if all of its bounded variables are ranked. If $\psi$ is a formula of $\mathcal{L}_{\infty}$, then $o(\psi)$ denotes the least upper bound of $\{\nu: \nu$ is the superscript of a quantified variable in $\psi\} \cup\left\{\nu+1\right.$ : some constant of the form $\boldsymbol{S}_{\nu, F, e}$ or $\boldsymbol{H}_{\nu, F}$ appears in $\left.\psi\right\}$. For every constant $c=C_{\lambda}^{F}$ above, we let $o(c)=o(\emptyset \in C)$. Also, for all $\psi \in \mathcal{L}_{\infty}$, define

$$
\operatorname{rk}(\psi)=\omega_{1}^{C K} \cdot u(\psi)+\omega^{2} \cdot o(\psi)+\omega \cdot r(\psi)+n(\psi),
$$

where $u(\psi)$ is the number of unranked quantifiers in $\psi, r(\psi)$ is the number of ranked quantifiers in $\psi$, and $n(\psi)$ is the number of connectives in $\psi$.

3.4. The forcing relation. The definition of the forcing relation is standard; for further details consult Mon. One can show (by transfinite induction) that if $p \in \boldsymbol{P}$ and $\psi \in \mathcal{L}_{\infty}$, then $p \Vdash \psi$ if and only if whenever $G$ is a sufficiently generic filter such that $p \in G$ and $\mathcal{M}_{\infty}$ is the model obtained from $G$, then $\mathcal{M}_{\infty} \vDash \psi$.

3.5. Retaggings in $\boldsymbol{P}$. We now introduce the notion of a retagging. This notion will play a significant role throughout the rest of Section 3, and we will define other notions of retaggings later on. For now, our definition of retagging is similar to that of Steel [Ste78] and Montalbán [Mon, and, as a result, both the statements and proofs of the results in this section are similar to those found in Section 2.5 of Mon.

Definition 3.2. Let $p, p^{*} \in \boldsymbol{P}, F \subset_{f} \omega$, and $\mu \in \omega_{1}^{C K}$ be given. Then $p^{*}$ is a $\mu-F$-absolute retagging of $p$, and we write $\operatorname{Ret}\left(\mu, F, p, p^{*}\right)$ if the following three conditions are satisfied:

(1) $T^{p}=T^{p^{*}}, F \subseteq \operatorname{dom}\left(f^{p}\right), f^{p}\left\lceil F=f^{p^{*}} \mid F\right.$.

(2) $\left(\forall \sigma \in T^{p}\right)\left[h^{p}(\sigma)<\mu \Rightarrow h^{p^{*}}(\sigma)=h^{p}(\sigma)\right]$.

(3) $\left(\forall \sigma \in T^{p}\right)\left[h^{p}(\sigma) \geq \mu \Rightarrow h^{p^{*}}(\sigma) \geq \mu\right]$.

It can be shown that, for a fixed subset $F \subset_{f} \omega$ and ordinal $\mu<\omega_{1}^{C K}$, we have that $\operatorname{Ret}(\mu, F, \cdot, \cdot)$ is an equivalence relation on $\boldsymbol{P}$. The intuition behind the following lemma is that $\operatorname{Ret}\left(\mu, F, p, p^{*}\right)$ holds only if $p, p^{*} \in \boldsymbol{P}$ are indistinguishable inside $M_{\mu, F}$.

Lemma 3.3 (쯔, Lemma 2.4]). Let $\psi$ be a ranked formula in $\mathcal{L}_{F}$, and let $p, p^{*} \in$ P. Then,

$$
\operatorname{Ret}\left(\omega \cdot \operatorname{rk}(\psi), F, p, p^{*}\right) \quad \Rightarrow \quad\left(p \Vdash \psi \Leftrightarrow p^{*} \Vdash \psi\right) .
$$

The following lemma is crucial to the proof of Lemma 3.3. It is also crucial to the proof of Theorem 3.1

Lemma 3.4 ([Mon, Lemma 2.5]). Let $p^{*}$ be an $\omega \cdot \beta-F$-absolute retagging of $p \in \boldsymbol{P}$ and suppose that $\gamma<\beta$ and $q \leq p$. Then, there exists $q^{*} \leq p^{*}$ such that $\operatorname{Ret}\left(\omega \cdot \gamma, F, q, q^{*}\right)$. See Figure 2.

Proof. We construct $q^{*} \in \boldsymbol{P}$ as follows. First, set $T^{q^{*}}=T^{q}$. Secondly, set $f^{q^{*}}(i)=$ $f^{q}(i)$, for all $i \in F$, and $f^{q^{*}}(i)=f^{p^{*}}(i)$, for all $i \in \operatorname{dom}\left(f^{p^{*}}\right) \backslash F$. Next, let $h^{q^{*}} \supseteq h^{p^{*}}$ be such that $h^{q^{*}}(\sigma)=\infty$ for all $\sigma \in T^{q^{*}} \backslash T^{p^{*}}, \sigma \subseteq f^{q^{*}}(i), i \in F$. Also, define $h^{q^{*}}(\sigma)=h^{q}(\sigma)$ whenever $h^{q}(\sigma)<\omega \cdot \gamma$, and set $h^{q^{*}}(\sigma)=\omega \cdot \gamma+|\sigma|_{Q}$, for all $\sigma \in Q$, 


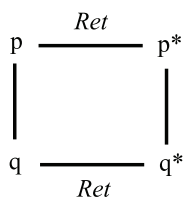

FiguRE 2

where $Q \subseteq T^{q^{*}}$ is the set of nodes in $T^{q^{*}}$ on which $h^{q^{*}}$ is currently undefined. Finally, let $g^{q^{*}} \supseteq g^{p^{*}}$ be such that $g^{q^{*}}(\sigma)=0$, for all $\sigma \in T_{\infty}^{q^{*}} \backslash T_{\infty}^{p^{*}}$.

One can check that $q^{*} \in \boldsymbol{P}, q^{*} \leq p^{*}$, and $\operatorname{Ret}\left(\omega \cdot \gamma, F, q, q^{*}\right)$.

The proof of Lemma 3.3 (above) is exactly the same as that of [Mon, Lemma 2.4].

Define $P_{\beta}=\left\{p \in \boldsymbol{P}: \operatorname{ran}\left(h^{p}\right) \subseteq \beta \cup\{\infty\}\right\}$. By Lemma 3.3, it follows that if $\psi=\neg \varphi$ is of rank $\beta$ and $p \in P_{\omega \cdot \beta}$, then $p \Vdash \neg \varphi$ if for every $q \in P_{\omega \cdot \beta}, q \leq p$, we have that $q \nVdash \varphi$. From this, we can conclude the following corollary (by transfinite induction on $\beta$ ).

Corollary 3.5 ( Mon, Corollary 2.6]). For a formula $\psi$ of rank $\beta, 0^{(\beta)}$ can decide whether or not $p \Vdash \psi$ uniformly in $\psi, p$, and $\beta$.

The first major application of Lemmas 3.3 and 3.4 is in the proof of the following lemma, a corollary of which says that our generic model $\mathcal{M}_{\infty}$ does not satisfy $A B W_{0}$.

Lemma 3.6 ([Mon, Lemma 2.7]). For every $F \subset_{f} \omega$, we have that

$$
M_{F} \cap\left[T^{G}\right]=\left\{\alpha_{i}^{G}: i \in F\right\} .
$$

Proof. Suppose, for a contradiction, that $S=S_{\nu, F, e} \in \mathcal{M}_{F}$ is a path through $T^{G}$ that is different from $\alpha_{i}^{G}$, for $i \in F$. Then there exists $\sigma \subset S,|\sigma|>1$, such that $\sigma$ is not an initial segment of $\alpha_{i}^{G}, i \in F$. Now, let $p \in G$ be such that $\operatorname{dom}\left(f^{p}\right) \supseteq F$, $\sigma \in T^{p}$, and

$$
p \Vdash \boldsymbol{S} \in[\boldsymbol{T}] \& \boldsymbol{\sigma} \subseteq \boldsymbol{S} \& \forall i \in F\left(\boldsymbol{\sigma} \nsubseteq \boldsymbol{\alpha}_{i}\right) .
$$

Now, let $\beta$ be greater than $\omega$ times the rank of $\psi$, and large enough so that $p \in P_{\beta}$. Since $S$ is a path in $T^{G}$, it follows that $h^{G}(\sigma)=\infty$, and thus $h^{p}(\sigma)=\infty$. We will construct a $p^{*} \in \boldsymbol{P}$ such that $\operatorname{Ret}\left(\beta, F, p, p^{*}\right)$ and $h^{p^{*}}(\sigma) \in \omega_{1}^{C K}$. To define $p^{*}$, all we need to do is change the values of $h^{p}(\tau), \tau \supseteq \sigma$, to ordinals in $\omega_{1}^{C K}$ that are greater than $\beta$.

Now, by Lemma 3.3 , we have that

$$
p^{*} \Vdash \boldsymbol{S} \in[\boldsymbol{T}] \& \sigma \subseteq \boldsymbol{S} \& \forall i \in F\left(\boldsymbol{\sigma} \nsubseteq \boldsymbol{\alpha}_{i}\right),
$$

a contradiction since $\sigma$ is in the well-founded part of $T^{G^{*}}$, for any generic filter $G^{*}$ extending $p^{*}$.

The following corollary says that our generic model $\mathcal{M}_{\infty}$ does not satisfy $A B W_{0}$. The reason for this, informally speaking, is that the set

$$
\left\{X: X \in\left[T^{G}\right]\right\}
$$

has no accumulation point in $M_{\infty}$. 
Before we state and prove the corollary, however, we require some elementary definitions. Recall (from the proof of Theorem 2.1) that

$$
V=\left\{X \in 2^{\omega}:\left(\exists^{\infty} n\right)[X(n)=1]\right\}
$$

we will refer to $V \subset 2^{\omega}$ as the set of irrational numbers, and the complement of $V$ (in $2^{\omega}$ ) as the set of rational numbers. Note that there is a natural computable homeomorphism $Z: V \rightarrow \omega^{\omega}$, such that $Z(X)(n), X \in V, n \in \omega$, is equal to the number of 0 s between the $n^{\text {th }}$ and $(n+1)^{\text {th }} 1$ s appearing in $X \in V$. Note also that for every $X \in V, Z(X) \equiv_{T} X$.

\section{Corollary 3.7.}

$$
\mathcal{M}_{\infty} \not \models \mathrm{ABW}_{0} .
$$

Proof. Let $A(X)$ be the bounded arithmetic formula of a single free set variable $X$ such that

$$
A(X) \equiv(X \in V) \wedge\left(Z(X) \in\left[T^{G}\right]\right) .
$$

Note that $A(X)$ is a $\Pi_{2}^{0, T^{G}}$ predicate, and therefore defines a $G_{\delta}$ set of reals.

First, note that $A(X)$ has infinitely many solutions in $M_{\infty}$ of the form $Z^{-1}\left(\alpha_{i}^{G}\right), i \in \omega$. Next, we will show that $\{X: A(X)\}$ has no accumulation point in $M_{\infty}$.

The first step in proving that $\{X: A(X)\}$ has no accumulation point in $M_{\infty}$ is to show that such an accumulation point cannot be rational. To see why this is the case, note that by condition (8b) and the genericity of $G \subseteq \boldsymbol{P}$, it follows that for any given node $\sigma \in T^{G}$ there exists a number $n_{\sigma} \in \omega$ such that for every $p \in G$ and every $\tau \in T^{G}$ such that $\tau \supseteq \sigma k, k \geq n_{\sigma}$, we have that $g^{p}(\tau)=0$ whenever it is defined. Therefore, if $X \in 2^{\omega}$ is an accumulation point of $\left\{X \in M_{\infty}: A(X)\right\}$, then $X$ must be irrational (i.e. $Z(X) \in \omega^{\omega}$ is defined).

Now, since $\left[T^{G}\right] \subseteq \omega^{\omega}$ and $Z^{-1}\left(\left[T^{G}\right]\right) \subseteq 2^{\omega}$ are closed sets, by the previous paragraph we have that every accumulation point of $\{X: A(X)\}$ must live inside $Z^{-1}\left(\left[T^{G}\right]\right)$ (but not necessarily inside $M_{\infty} \cap Z^{-1}\left(\left[T^{G}\right]\right)$ ). Furthermore, by Lemma 3.6. to show that $\{X: A(X)\}$ has no accumulation point in $M_{\infty}$, it suffices to show that for every $i \in \omega, Z^{-1}\left(\alpha_{i}^{G}\right) \in 2^{\omega}$ is not an accumulation point of $\left\{X \in M_{\infty}\right.$ : $A(X)\}$. We prove this by contradiction.

Suppose, for a contradiction, that there exists some $i \in \omega$ such that $Z^{-1}\left(\alpha_{i}^{G}\right) \in$ $2^{\omega}$ is an accumulation point of the set $\{X: A(X)\}$, and let $p \in \boldsymbol{P}$ be such that $i \in$ $\operatorname{dom}\left(f^{p}\right)$. Now, by genericity of $\boldsymbol{G} \subseteq \boldsymbol{P}$, let $q \leq p, q \in \boldsymbol{P}$, be such that all $\sigma \in T_{\infty}^{q}$ such that $g^{q}(\sigma)=1$ are incomparable with $f^{q}(i) \in T_{\infty}^{q}$. By our condition (9) in Section 3.2, it follows that $Z^{-1}\left(\alpha_{i}^{G}\right) \in 2^{\omega}$ is not an accumulation point for the set $\{X: A(X)\}$, because $\left[T^{G}\right] \cap\left[f^{q}(i)\right] \cap M_{\infty}=\left\{\alpha_{i}^{G}\right\}$, and thus $\alpha_{i}^{G}$ is isolated in $\left[T^{G}\right]$, from the point of view of $M_{\infty}$.

Note that Theorem 2.4 above implies that we could not have produced a simpler formula (in terms of quantifiers) for $A(X)$. In other words, Corollary 3.7 is sharp in terms of the quantifier complexity of $A(X)$.

Following [Ste78, Mon], for all $F \subset_{f} \omega$, we say that a formula $\psi \in \mathcal{L}_{\infty}$ is $\Sigma$-over$\mathcal{L}_{F}$ if it is built up from ranked, $F$-restricted formulas using $\wedge, \forall n$, and $\exists X$. For any formula $\psi \in \mathcal{L}_{\infty}$ and $\mu<\omega_{1}^{C K}, \psi^{\mu}$ is the result of replacing " $X$ " by " $X^{\mu}$ ", for every unranked variable $X$. Note that if $F \subset_{f} \omega, \psi$ is $\Sigma$-over- $\mathcal{L}_{F}, \mu<\omega_{1}^{C K}$, and $\mu>o(\boldsymbol{d})$ for any constant $\boldsymbol{d}$ appearing in $\psi$, then we have that $\psi^{\mu} \Rightarrow \psi$.

The proof of the following lemma is very similar to the one given in Mon. 
Lemma 3.8 ([Mon, Lemma 2.9]).

(1) Let $p \in \boldsymbol{P}, p \Vdash \psi$, where $\psi \in \mathcal{L}_{\infty}$ is $\Sigma$-over- $\mathcal{L}_{F}$, and $F \subset_{f} \omega$. Then $\left(\exists \mu<\omega_{1}^{C K}\right)(\forall \rho)\left[\mu \leq \rho<\omega_{1}^{C K} \Rightarrow\right.$ $\left.p \Vdash \psi^{\rho}\right]$.

(2) $M_{F} \vDash \Sigma_{1}^{1}-\mathrm{AC}_{0}$, and hence $M_{F}$ is hyperarithmetically closed. Moreover, $M_{F}=\operatorname{HYP}\left(T \oplus \bigoplus_{i \in F} \alpha_{i}^{G}\right)$.

3.6. Automorphisms of $\boldsymbol{P}$. Let $\pi: \omega \rightarrow \omega$ be an automorphism (i.e. permutation) of $\omega$. Then $\pi$ induces an automorphism $\hat{\pi}$ of $\boldsymbol{P}$ as follows: $T^{\hat{\pi}(p)}=T^{p}, h^{\hat{\pi}(p)}=$ $h^{p}, g^{\hat{\pi}(p)}=g^{p}$, and $f^{\hat{\pi}(p)}(\pi(i))=f^{p}(i), i \in \omega$. Automorphisms of the form $\hat{\pi}$, $\pi: \omega \rightarrow \omega$ a permutation, play a significant role throughout the rest of this section. Given $\varphi \in \mathcal{L}_{\infty}$, let $\pi(\psi)$ be the formula obtained from $\psi$ by replacing $\boldsymbol{\alpha}_{i}$ by $\boldsymbol{\alpha}_{\pi(i)}$ for every $i \in \omega$.

The following lemma is proved by induction on the rank of $\psi \in \mathcal{L}_{\infty}$.

Lemma 3.9 ([Mon, Lemma 2.10]). Let $\pi$ be a permutation of $\omega$, let $p \in \boldsymbol{P}$, and let $\psi \in \mathcal{L}_{\infty}$. Then

$$
p \Vdash \psi \Leftrightarrow \hat{\pi}(p) \Vdash \pi(\psi) .
$$

Remark. As in Mon, we shall mainly use automorphisms (of $\boldsymbol{P}$ ) of the form $\hat{\pi}$ in the following way. Let $F \subset_{f} \omega$, and let $K \subset_{f} \omega$ be such that $F \cap K=\emptyset$. Also, suppose that $p \in \boldsymbol{P}$ has $\operatorname{dom}\left(f^{p}\right) \subseteq F$, that $\psi$ has constants in $\mathcal{L}_{F}$, and that $r \in \boldsymbol{P}$ is such that $r \leq p$ and $r \Vdash \psi$. Now, via an automorphism of $\boldsymbol{P}$, we can replace $r$ by a condition $\hat{\pi}(r)$ such that $\hat{\pi}(r) \leq p, \hat{\pi}(r) \Vdash \psi$, and $\operatorname{dom}\left(f^{\hat{\pi}}(r)\right) \cap K$. In other words, we could replace $r$ by $\hat{\pi}(r)$ if necessary to guarantee that $\operatorname{dom}(r) \cap K$ is disjoint from $F \subset_{f} \omega$.

We now introduce a stronger notion of retagging, which we shall use throughout the rest of this section.

Definition 3.10. Let $p, p^{*} \in \boldsymbol{P}, F \subset_{f} \omega$, and $\mu \in \omega_{1}^{C K}$ be given. Then $p^{*}$ is a good $\mu-F-$ absolute retagging of $p$, and we write $\underline{\operatorname{Ret}}\left(\mu, F, p, p^{*}\right)$ if the following conditions are satisfied:

(1) $T^{p}=T^{p^{*}}$ and $f^{p} \uparrow F=f^{p^{*}}\lceil F$.

(2) $\left(\forall \sigma \in T^{p}\right)\left[h^{p}(\sigma)<\mu \Rightarrow h^{p^{*}}(\sigma)=h^{p}(\sigma)\right]$.

(3) $\left(\forall \sigma \in T^{p}\right)\left[h^{p}(\sigma) \geq \mu \Rightarrow h^{p^{*}} \geq \mu\right]$.

(4) $T_{\infty}^{p} \subseteq T_{\infty}^{p^{*}}$.

(5) $\left(\forall \sigma \in T_{\infty}^{p}\right)\left[g^{p}(\sigma)=1 \Rightarrow g^{p^{*}}(\sigma)=1\right]$.

We also define $\underline{\operatorname{Ret}}_{F}\left(\mu, F, p, p^{*}\right)$ exactly as $\underline{\operatorname{Ret}}\left(\mu, F, p, p^{*}\right)$, except that we also require $F \subseteq \operatorname{dom}\left(f^{p}\right)$ in condition (1). Note that $\underline{\operatorname{Ret}}$ and $\underline{\operatorname{Ret}}_{F}$ are not equivalence relations (because of condition (5)).

We now prove some retagging lemmas concerning $\underline{\operatorname{Ret}}$ and $\underline{\operatorname{Ret}}_{F}$. These lemmas are similar in spirit to Lemmas 3.3 and 3.4 above (or [Mon, Lemma 2.4, Lemma $2.5])$.

Lemma 3.11. Let $\psi$ be a ranked formula in $\mathcal{L}_{F}$, and let $p, p^{*} \in \boldsymbol{P}$. Then,

$$
\underline{\operatorname{Ret}}_{F}\left(\omega \cdot \operatorname{rk}(\psi), F, p, p^{*}\right) \quad \Rightarrow \quad\left(p \Vdash \psi \Leftrightarrow p^{*} \Vdash \psi\right) .
$$

Note that Lemma 3.3 is a particular case of Lemma 3.11. It depends heavily upon Corollary 3.13 and Lemma 3.14 below. First, however, we prove Lemma 3.12 which is a stronger version of Corollary 3.13 Lemma 3.12 will play a major role in the proof of Lemma 3.15 below. 
Lemma 3.12. Suppose that $F \subset_{f} \omega, \underline{\operatorname{Ret}}\left(\omega \cdot \beta, F, p, p^{*}\right)$, and that $\gamma<\beta$ and $q \leq p$. Then, there exists $q^{*} \leq p^{*}$ such that $\underline{\operatorname{Ret}}\left(\omega \cdot \gamma, F, q, q^{*}\right)$.

Proof. We construct $q^{*} \in \boldsymbol{P}$ as follows. First, define $T^{q^{*}}=T^{q}$. Secondly, set $f^{q^{*}}(i)=f^{q}(i)$, for all $i \in F \cap \operatorname{dom}\left(f^{q}\right)$, and $f^{q^{*}}(i)=f^{p^{*}}(i)$, for all $i \in \operatorname{dom}$ $\left(f^{p^{*}}\right) \backslash F$. Next, we define $h^{q^{*}}: T^{q^{*}} \rightarrow \omega_{1}^{C K} \cup\{\infty\}$ as follows.

(a) Let $h^{q^{*}}(\sigma)=h^{p^{*}}(\sigma)$, for all $\sigma \in T^{p^{*}}$.

(b) Let $h^{q^{*}}(\sigma)=\infty$, for all $\sigma \in T^{q^{*}} \backslash T^{p^{*}}$ such that $h^{q}(\sigma)=\infty$.

(c) Let $h^{q^{*}}(\sigma)=h^{q}(\sigma)$, whenever $\sigma \in T^{q^{*}} \backslash T^{p^{*}}$ is such that $h^{q}(\sigma)<\omega \cdot \gamma$.

(d) Let $h^{q^{*}}(\sigma)=\omega \cdot \gamma+|\sigma|_{Q}$ for all $\sigma \in Q$, where $Q \subseteq T^{q^{*}} \backslash T^{p^{*}}$ is the set of nodes in $T^{q^{*}} \backslash T^{p^{*}}$ not covered by cases (a)-(c) above.

Lastly, we define $g^{q^{*}}: T_{\infty}^{q^{*}} \rightarrow\{0,1\}$ as follows.

(i) For all $\sigma \in T^{p^{*}}$, let $g^{q^{*}}(\sigma)=0$, if either $g^{p^{*}}(\sigma)=0$, or else $g^{p}(\sigma)=1$ and $g^{q}(\sigma)=0$. Let $g^{q^{*}}(\sigma), \sigma \in T^{p^{*}}$, be equal to 1 otherwise.

(ii) Let $g^{q^{*}}(\sigma)=1$, for all $\sigma \in T^{q^{*}} \backslash T^{p^{*}}$ such that $g^{q}(\sigma)=1$.

(iii) Let $g^{q^{*}}(\sigma)=0$, for all $\sigma \in T^{q^{*}} \backslash T^{p^{*}}$ such that $g^{q}(\sigma)=0$.

One can verify that, by the construction of $q^{*}$, we have that $q^{*} \in \boldsymbol{P}, q^{*} \leq p^{*}$, and $\underline{\operatorname{Ret}}\left(\omega \cdot \gamma, F, q, q^{*}\right)$, as required.

The statement of the following corollary is the same as that of the previous lemma, except that we replace $\underline{\operatorname{Ret}}$ with $\underline{\operatorname{Ret}}_{F}$. Moreover, it follows immediately from Lemma 3.12

Corollary 3.13. Suppose that $F \subset_{f} \omega, \underline{\operatorname{Ret}}_{F}\left(\omega \cdot \beta, F, p, p^{*}\right)$, and that $\gamma<\beta$ and $q \leq p$. Then, there exists $q^{*} \leq p^{*}$ such that $\underline{\operatorname{Ret}}_{F}\left(\omega \cdot \gamma, F, q, q^{*}\right)$.

The next lemma is the counterpart to Lemma 3.12 above, except that it can only be verified with $\underline{R e t}_{F}$ in place of $\underline{\text { Ret }}$.

Lemma 3.14. Suppose that $F \subset_{f} \omega, \underline{\operatorname{Ret}}_{F}\left(\omega \cdot \beta, F, p, p^{*}\right)$, and that $\gamma<\beta$ and $q^{*} \leq p^{*}$. Then, there exists $q \leq p$ such that $\underline{\operatorname{Ret}}_{F}\left(\omega \cdot \gamma, F, q, q^{*}\right)$.

Proof. We construct $q \in \boldsymbol{P}$ as follows. First, define $T^{q}=T^{q^{*}}$. Secondly, set $f^{q}(i)=f^{q^{*}}(i)$, for all $i \in F$, and $f^{q}(i)=f^{p}(i)$, for all $i \in \operatorname{dom}\left(f^{p}\right) \backslash F$. Next, we define $h^{q}: T^{q} \rightarrow \omega_{1}^{C K} \cup\{\infty\}$ as follows.

(a) $h^{q}(\sigma)=h^{p}(\sigma)$, for all $\sigma \in T^{p}$.

(b) $h^{q}(\sigma)=\infty$, for all $\sigma \in T^{q} \backslash T^{p}$ such that $h^{q^{*}}(\sigma)=\infty$ and $h^{p}\left(\tau_{\sigma}\right)=\infty$, where $\tau_{\sigma} \in T^{p}$ is the longest initial segment of $\sigma$ on $T^{p}$.

(c) $h^{q}(\sigma)=h^{q^{*}}(\sigma)$, whenever $\sigma \in T^{q} \backslash T^{p}$ and $h^{q^{*}}(\sigma)<\omega \cdot \gamma$.

(d) $h^{q}(\sigma)=\omega \cdot \gamma+|\sigma|_{Q}$ for all $\sigma \in Q$, where $Q \subseteq T^{q} \backslash T^{p}$ is the set of nodes in $T^{q} \backslash T^{p}$ not covered by cases (a)-(c) above.

Lastly, we define $g^{q}: T_{\infty}^{q} \rightarrow\{0,1\}$ as follows.

(i) For all $\sigma \in T^{p}$, let $g^{q}(\sigma)=0$, if either $g^{p}(\sigma)=0$, or else $g^{p^{*}}(\sigma)=1$ and $g^{q^{*}}(\sigma)=0$. Let $g^{q}(\sigma), \sigma \in T^{p}$, be equal to 1 otherwise.

(ii) Let $g^{q}(\sigma)=1$, for all $\sigma \in T^{q} \backslash T^{p}$ such that $g^{q^{*}}(\sigma)=1$ and there is some $\tau \subset \sigma, \tau \in T^{p}$, such that $g^{p}(\tau)=1$ but $g^{q}(\tau)=0$ via case (i) above.

(iii) Let $g^{q^{*}}(\sigma)=0$, for all $\sigma \in T^{q^{*}} \backslash T^{p^{*}}$ not covered by case (ii) above.

One can verify that, by condition (8a) in Section 3.2 and our construction of $q$ above, we have that $q \in \boldsymbol{P}, q \leq p$, and $\underline{\operatorname{Ret}}_{F}\left(\omega \cdot \gamma, F, q, q^{*}\right)$, as required. 
The following key lemma (Lemma 3.15) will play a major role in the proof of Theorem 3.17 (below), which says that $M_{\infty}$ satisfies $\Delta_{1}^{1}-\mathrm{CA}_{0}$. Its statement is similar to that of [Mon, Lemma 2.12], but our proof depends heavily on the previous two (new) lemmas and corollary.

Lemma 3.15. Let $F \subset_{f} \omega$ and $\psi \in \mathcal{L}_{\infty}$ be a $\Sigma$-over- $\mathcal{L}_{F}$ sentence. Suppose also that $\nu=\operatorname{rk}\left(\psi^{\mu}\right)$, where $\mu<\omega_{1}^{C K}$. Then,

$$
\underline{\operatorname{Ret}}_{F}\left(\omega \nu+\omega^{2}, F, p, p^{*}\right) \& \operatorname{dom}\left(f^{p}\right)=F \quad \Rightarrow \quad\left(p \Vdash \psi^{\mu} \Rightarrow p^{*} \Vdash \psi^{\mu}\right) .
$$

(Note that $\psi^{\mu}$ is not necessarily in $\mathcal{L}_{F}$, because it may have quantifiers of the form $\exists X^{\mu}$.)

Proof. The proof is by induction on the number $k \in \omega$ of steps needed to build $\psi$ from ranked, $F$-restricted formulas; we will show that the formula holds with " $\omega \nu+\omega 2 k$ " replacing " $\omega \nu+\omega^{2}$ ". The case $k=0$ follows directly from Lemma 3.3 above. All of the cases follow easily from the induction hypothesis, except when $\psi$ is of the form $\exists X \varphi(X)$. In this case we must show that $\left(\forall q^{*} \leq p^{*}\right)\left(\exists r^{*} \leq q^{*}\right)(\exists \boldsymbol{S} \in$ $\left.C_{\mu}\right)\left[r^{*} \Vdash \varphi^{\mu}(\boldsymbol{S})\right]$.

Now, let $q^{*} \leq p^{*}$ be given. By Lemma 3.14, there exists $q \leq p$ such that $\underline{\operatorname{Ret}}_{F}\left(\omega \nu+\omega(2 k+1), F, q, q^{*}\right)$. Moreover, the proof of Lemma 3.14 produces such a $q \leq p$ with $\operatorname{dom}\left(f^{q}\right)=F$. Since $p \Vdash \psi^{\mu}$, there exists $r \leq q$ and $\boldsymbol{S} \in C_{\mu}$ such that $r \Vdash \varphi^{\mu}(\boldsymbol{S})$. Choose $H \subset_{f} \omega$ such that $\boldsymbol{S} \in C_{\mu}^{F \cup H}$, $\operatorname{dom}\left(f^{r}\right)=F \cup H$, and $F \cap H=\emptyset$. Using an automorphism of $\boldsymbol{P}$ if necessary, we can assume without loss of generality that $H \cap \operatorname{dom}\left(f^{q^{*}}\right)=\emptyset$. Then we have that $\underline{\operatorname{Ret}}\left(\omega \nu+\omega(2 k+1), F \cup H, q, q^{*}\right)$, and by Lemma 3.12, there exists $r^{*} \leq q^{*}$ such that $\underline{\operatorname{Ret}}_{F \cup H}\left(\omega \nu+\omega 2 k, F \cup H, r, r^{*}\right)$. Finally, we can apply the induction hypothesis to conclude that $r^{*} \Vdash \varphi^{\mu}(\boldsymbol{S})$.

3.7. $\mathcal{M}_{\infty}$ satisfies $\Delta_{1}^{1}-\mathrm{CA}_{0}$. Before we can prove that $M_{\infty}$ satisfies $\Delta_{1}^{1}-\mathrm{CA}_{0}$, we require the following definition.

Definition 3.16. Suppose that $T^{\prime} \subseteq_{f} T^{G}$ and $g: T^{\prime} \rightarrow \omega_{1}^{C K} \cup\{\infty\}$. We say that $g$ is $\nu$-good if

$$
\left(\forall \sigma \in T^{\prime}\right)\left[\left(h^{G}(\sigma)<\nu \Rightarrow g(\sigma)=h^{G}(\sigma)\right) \&\left(h^{g}(\sigma) \geq \nu \Rightarrow g(\sigma) \geq \nu\right)\right] .
$$

Note that deciding whether or not $g$ is $\nu$-good is hyperarithmetic in $g, T^{G}$, and $\nu$, since it requires at most $(\nu+\omega)$-many Turing jumps of $T^{G}$.

We are now ready to prove that $M_{\infty}$ satisfies $\Delta_{1}^{1}-\mathrm{CA}_{0}$.

\section{Theorem 3.17.}

$$
M_{\infty} \vDash \Delta_{1}^{1}-\mathrm{CA}_{0} .
$$

Proof. The proof is similar to that of [Mon, Lemma 2.14], with a few modifications.

Let $\varphi(n), \psi(n)$ be $\Sigma$-over- $\mathcal{L}_{F}$ with only $n$ free, $F \subset_{f} \omega$, and such that

$$
M_{\infty} \vDash(\forall n)[\psi(n) \Leftrightarrow \neg \varphi(n)] .
$$

We need to show that there exists $D \in M_{\infty}$ such that

$$
M_{\infty} \vDash(\forall n)[\psi(n) \Leftrightarrow n \in D] .
$$

Let $p \in G$ be such that $p \Vdash(\forall n)[\psi(n) \Leftrightarrow \neg \varphi(n)]$. By enlarging $F \subset_{f} \omega$ (if necessary) and taking an extension $p^{\prime} \leq p$, we can assume without any loss of generality that $\operatorname{dom}\left(f^{p}\right)=F$. By Lemma 3.8 there exists $\mu<\omega_{1}^{C K}$ such that

$$
p \Vdash(\forall n)\left[\psi^{\mu}(n) \vee \varphi^{\mu}(n)\right]
$$


and $\mu>o(\boldsymbol{S})$, for any constant $\boldsymbol{S}$ occurring in either $\psi$ or $\varphi$. Fix $\nu<\omega_{1}^{C K}$ such that $p \in P_{\nu}$ and for all $n \in \omega$ we have that $\operatorname{rk}\left(\varphi^{\mu}(\boldsymbol{n}) \Leftrightarrow \neg \psi^{\mu}(\boldsymbol{n})\right)<\nu$. We are now ready to define the set $D \in M_{\infty}, D \subseteq \omega$ described above.

Let $d \in D$ if and only if there exists $q \in P_{\omega \nu+\omega^{2}+\omega 2}, q \leq p$, such that

(1) $q \Vdash \psi^{\mu}(\boldsymbol{d})$;

(2) $T^{q} \subset T^{G}$;

(3) $h^{q}$ is $\omega \nu+\omega^{2}+\omega 2$-good;

(4) $(\forall i \in F)\left[f^{q}(i)\right.$ is the longest initial segment of $\alpha_{i}^{G}$ on $\left.T^{q}\right]$.

We will show that, for every $d \in \omega$, we have that $d \in D$ if and only if $\neg \varphi(d)$ holds.

Note that $D \subseteq \omega$ is hyperarithmetic in $T \oplus \bigoplus_{i \in F} \alpha_{i}^{G}$. Furthermore, since $M_{F}=$ $\operatorname{HYP}\left(T \oplus \bigoplus_{i \in F} \alpha_{i}^{G}\right)$, we have that $D \in M_{F} \subseteq M_{\infty}$.

Now, assume that $d \in \omega$ is such that $\neg \varphi(d)$. We will show that $d \in D$. Since $\varphi$ is $\Sigma$-over- $\mathcal{L}_{F}$, by definition of $\mu, \neg \varphi^{\mu}(d)$ holds. Let $q \in G, q \leq p$, be such that $q \Vdash \neg \varphi^{\mu}(\boldsymbol{d})$, and hence $q \Vdash \psi^{\mu}(\boldsymbol{d})$. By construction, $q$ satisfies conditions (1)-(4) above, but $q$ may not be in $P_{\omega \nu+\omega^{2}+\omega 2}$. To fix this issue, define $q^{*} \in \boldsymbol{P}$ as follows. Begin by setting $T^{q}=T^{q^{*}}, f^{q}=f^{q^{*}} \uparrow F$, and $h^{q^{*}} \supseteq h^{p}$. Then, for all $\sigma \in T^{q^{*}} \backslash T^{p}$, define $h^{q^{*}}(\sigma)=\infty$, whenever $h^{q}(\sigma) \geq \omega \nu+\omega^{2}+\omega 2$, and $h^{q^{*}}(\sigma)=h^{q}(\sigma)$ otherwise. Finally, set $g^{q^{*}}(\sigma)=1$ for all $\sigma \in T_{\infty}^{q^{*}}$ such that $\sigma \in T_{\infty}^{q}$ and $g^{q}(\sigma)=1$, and set $g^{q^{*}}(\sigma)=0$ otherwise. By our construction of $q^{*}$, it follows easily that $q^{*} \in P_{\omega \nu+\omega^{2}+\omega 2}, q^{*} \leq p$, and that $q^{*}$ satisfies (2)-(4) above. To see that $q^{*}$ also satisfies condition (1), note that $\underline{\operatorname{Ret}}_{F}\left(\omega \nu+\omega^{2}+\omega 2, F, q, q^{*}\right)$. Hence, by definition of $q \leq p$ and Lemma 3.15 above, it follows that $q^{*}$ also satisfies (1). Hence, $q^{*}$ witnesses that $d \in D$.

Now, assume that $d \in \omega$ is such that $\varphi(d)$ holds. We will show that $d \notin D$. Let $r \leq p, r \in G$, be such that $r \Vdash \varphi(\boldsymbol{d})$, and thus $r \Vdash \varphi^{\mu}(\boldsymbol{d}) \wedge \neg \psi^{\mu}(\boldsymbol{d})$. Now, suppose for a contradiction that $d \in D$ and $q \in \boldsymbol{P}$ witnesses it. Via an automorphism of $\boldsymbol{P}$, we may assume without loss of generality that $\operatorname{dom}\left(f^{q}\right)=F \cup H, H \subset{ }_{f} \omega$, where $F \cap H=\emptyset$ and $H \cap \operatorname{dom}\left(f^{r}\right)=\emptyset$. Let $F_{q^{*}} \subset_{f} \omega$ denote $\operatorname{dom}\left(f^{q^{*}}\right)$, and let $F_{r^{*}} \subset_{f} \omega$ denote $\operatorname{dom}\left(f^{r^{*}}\right)$. Next (see Figure 3), we will define $q^{*} \leq q, r^{*} \leq r$, and $s^{*} \leq p$, such that

(i) $\underline{\operatorname{Ret}}_{F_{q^{*}}}\left(\omega \nu+\omega^{2}+\omega, F_{q^{*}}, q^{*}, s^{*}\right)$;

(ii) $\underline{\operatorname{Ret}}_{F_{r^{*}}}\left(\omega \nu+\omega^{2}+\omega, F_{r^{*}}, r^{*}, s^{*}\right)$.

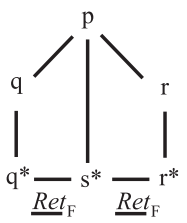

Figure 3

Now, since $r \Vdash \varphi^{\mu}(\boldsymbol{d})$ and $q \Vdash \psi^{\mu}(\boldsymbol{d})$, then by Lemma 3.15, it will follow that $s^{*} \Vdash \varphi^{\mu}(\boldsymbol{d}) \wedge \psi^{\mu}(\boldsymbol{d})$. However, since $s^{*} \leq p$ and $p \Vdash(\forall n)\left[\psi^{\mu}(n) \Leftrightarrow \neg \varphi^{\mu}(n)\right]$, we have a contradiction. All that is left to do is to construct $q^{*}, r^{*}$, and $s^{*}$.

We now construct a tree $T \supseteq T^{q} \cup T^{r}, T \subseteq 2^{<\omega}$, as follows. First, let $A \subseteq \omega^{<\omega}$ be the set of $\rho \in T_{\infty}^{q} \cup T_{\infty}^{r}$ such that either $g^{q}(\rho)=1$ or $g^{r}(\rho)=1$. Next, for every 
$\rho \in A$, let $\tau_{\rho} \in \omega^{<\omega}$ be any node such that $\tau_{\rho} \supset \rho,\left|\tau_{\rho}\right|=|\rho|+1$, and $\tau_{\rho} \notin T^{q} \cup T^{r}$. Now, let

$$
T=T^{q} \cup T^{r} \cup\left\{\tau_{\rho}: \rho \in A\right\} ;
$$

note that, by its construction, $T \subseteq \omega^{<\omega}$ is a tree. Let $R=\left\{\tau_{\rho}: \rho \in A\right\}=$ $T \backslash\left(T^{q} \cup T^{r}\right)$.

Define $q^{*}$ as follows.

(1) $T^{q^{*}}=T$.

(2) (i) $f^{q^{*}}(i)=f^{q}(i)$, for $i \in H$.

(ii) $f^{q^{*}}(i)=\alpha_{i}^{G}\lceil n$, where $n \in \omega$ is the largest number such that $\alpha_{i}^{G}\left\lceil n \in T^{q^{*}}\right.$, and $i \in F$.

(3) (a) $h^{q^{*}}(\tau)=h^{q}(\tau)$, for all $\tau \in T^{q}$.

(b) $h^{q^{*}}(\tau)=h^{r}(\tau)$, for all $\tau \in T^{r} \backslash T^{q}$ such that $h^{r}(\tau)<\omega \nu+\omega^{2}+\omega$.

(c) $h^{q^{*}}(\tau)=\infty$ if $(\exists i)\left[\tau \subseteq f^{q^{*}}(i)\right]$.

(d) $h^{q^{*}}(\tau)=\infty$ if $\tau \in R$, and $h^{q}\left(\sigma_{\tau}\right)=\infty$ where $\sigma_{\tau}$ is the longest initial segment of $\tau$ on $T^{q}$.

(e) $h^{q^{*}}(\tau)=\omega \nu+\omega^{2}+\omega+|\tau|_{Q}$, for all $\tau \in Q$, where $Q=\{\tau \in T$ : $\tau$ is not covered by cases (a), (b), or (c) above .

(4) $g^{q^{*}}(\sigma)=1$ for all $\sigma \in R$ such that $g^{q}(\tau)=1$, for some $\tau \subset \sigma, \tau \in T^{q}$; set $g^{q^{*}}(\sigma)=0$ otherwise.

By the constuction of $q^{*}$, it follows that $q^{*} \in \boldsymbol{P}, h^{q^{*}}$ is $\left(\omega \nu+\omega^{2}+\omega\right)$-good, and $q^{*} \leq q$.

We define $r^{*}$ as follows.

(1) $T^{r^{*}}=T$.

(2) (i) $f^{r^{*}}(i)=f^{r}(i)$, for $i \in \operatorname{dom}\left(f^{r}\right) \backslash F$.

(ii) $f^{r^{*}}(i)=\alpha_{i}^{G}\left\lceil n\right.$, where $n$ is the largest number such that $\alpha_{i}^{G}\lceil n \in$ $T$, for each $i \in F$.

(3) (a) $h^{r^{*}}(\tau)=\infty$, for all $\tau \in R$ such that $h^{r}\left(\sigma_{\tau}\right)=\infty$, where $\sigma_{\tau} \in T^{r}$ is the longest initial segment of $\tau$ on $T^{r}$.

(b) $h^{r^{*}}(\tau)=h^{G}(\tau)$, for all $\tau \in T$ not covered by case (a).

(4) $g^{r^{*}}(\sigma)=1$ for all $\sigma \in R$ such that $g^{r}(\tau)=1$, for some $\tau \subset \sigma, \tau \in T^{r}$; set $g^{r^{*}}(\sigma)=0$ otherwise.

It is not difficult to check that $r^{*} \in \boldsymbol{P}, r^{*} \leq r$, and $h^{r^{*}}$ is $\omega \nu+\omega^{2}+\omega-\operatorname{good}$.

Lastly, we construct $s^{*} \leq p$ as follows.

(1) $T^{s^{*}}=T$.

(2) (i) $f^{s^{*}}(i)=f^{q^{*}}(i)=f^{r^{*}}(i)$, for all $i \in F$.

(ii) $f^{s^{*}}(i)=f^{q^{*}}(i)$, for all $i \in \operatorname{dom}\left(f^{q^{*}}\right) \backslash F$.

(iii) $f^{s^{*}}(i)=f^{r^{*}}(i)$, for all $i \in \operatorname{dom}\left(f^{r^{*}}\right) \backslash F$.

(3) (a) $h^{s^{*}}(\tau)=h^{p}(\tau)$, for all $\tau \in T^{p}$.

(b) $h^{s^{*}}(\tau)=h^{q^{*}}(\tau)=h^{r^{*}}(\tau)=h^{G}(\tau)$, for all $\tau \in T^{s^{*}} \backslash T^{p}$ such that $h^{G}(\tau)<\omega \nu+\omega^{2}+\omega$.

(c) $h^{s^{*}}(\tau)=\infty$, for all $\tau \in T^{s^{*}} \backslash T^{p}$ such that either $h^{q^{*}}(\tau)=\infty$ or $h^{r^{*}}(\tau)=\infty$

(d) $h^{s^{*}}(\tau)=\omega \nu+\omega^{2}+\omega+|\tau|_{Q}$, for all $\tau \in Q$, where $Q=\left\{\tau \in T^{s^{*}}\right.$ : $\tau$ is not covered by cases (a), (b), or (c) above\}.

(4) $g^{s^{*}}(\sigma)=1$, for all $\sigma \in R$; set $g^{s^{*}}(\sigma)=0$ otherwise. 
It follows from the constructions of $q^{*}, r^{*}, s^{*}$ above that $q^{*}, r^{*}, s^{*} \in \boldsymbol{P}, q^{*} \leq q$, $r^{*} \leq r, s^{*} \leq p$, and that conditions (i)-(ii) above are satisfied, as required.

This completes the proof of Theorem [3.1. The following corollary says that Theorem 3.1 applies just as well to $\mathrm{G}_{\delta \sigma} \mathrm{ABW}_{0}$ in place of $\mathrm{ABW}_{0}$.

Corollary 3.18. $\Delta_{1}^{1}-\mathrm{CA}_{0}$ does not imply $\mathrm{G}_{\delta} \mathrm{ABW}_{0}, \mathrm{~F}_{\sigma \delta} \mathrm{ABW}_{0}$, or $\mathrm{G}_{\delta \sigma} \mathrm{ABW}_{0}$.

Proof. Let $B \in\left\{G_{\delta}, F_{\sigma \delta}, G_{\delta \sigma}\right\}$, and note that every $G_{\delta}$ set of reals is also a $B$ set of reals. Now, the predicate $A(X)$ that we defined in Corollary 3.7 above is $\Pi_{2}^{0, T^{G}}$ and hence corresponds to a $\left(G_{\delta}\right.$ and hence a) $B$ set of reals. It follows that our model $M_{\infty}$ above is a model of $\Delta_{1}^{1}-\mathrm{CA}_{0}$ but not a model of $\mathrm{B} A \mathrm{BW}_{0}$.

Corollary 3.19. $\mathrm{G}_{\delta} \mathrm{ABW}_{0}$ and $\mathrm{F}_{\sigma \delta} \mathrm{ABW}_{0}$ are strictly stronger than $\mathrm{ACA}_{0}$.

Proof. First of all, note that $\mathrm{F}_{\sigma \delta} \mathrm{ABW}_{0}$ implies $\mathrm{G}_{\delta} \mathrm{ABW}_{0}$ (since every $G_{\delta}$ set of reals is $F_{\sigma \delta}$ ), and via Theorem 2.4 above we know that $\mathrm{G}_{\delta} \mathrm{ABW}_{0}$ implies $\mathrm{ACA}_{0}$. It suffices to show that $A C A_{0}$ does not imply $G_{\delta} A B W_{0}$. This follows from the previous corollary and the fact that $\Delta_{1}^{1}-\mathrm{CA}_{0}$ implies $A C \mathrm{~A}_{0}$.

\section{ABW D DOES NOT IMPLY INDEC $_{0}$}

Our main goal in this section is to prove the following theorem.

Theorem 4.1. There is an $\omega$-model $\mathcal{N}$ that satisfies $\mathrm{ABW}_{0}$, but does not satisfy $\mathrm{INDEC}_{0}$. Therefore, $\mathrm{ABW}_{0}$ does not imply $\mathrm{INDEC}_{0}$.

We obtain the following immediate corollary.

Corollary 4.2. $\mathrm{G}_{\delta} \mathrm{ABW}_{0}, \mathrm{~F}_{\sigma \delta} \mathrm{ABW}_{0}$, and $\mathrm{G}_{\delta \sigma} \mathrm{ABW}_{0}$ do not imply $\mathrm{INDEC}_{0}$.

Proof. Let $\mathrm{B} \in\left\{\mathrm{G}_{\delta} \mathrm{ABW} \mathrm{W}_{0}, \mathrm{~F}_{\sigma \delta} \mathrm{ABW}_{0}, \mathrm{G}_{\delta \sigma} \mathrm{ABW}_{0}\right\}$, and note that $\mathrm{ABW}_{0}$ implies $\mathrm{B}$. Therefore, any model that satisfies $A B W_{0}$ must also satisfy $B$. Thus, $\mathcal{N}$ satisfies $B$ but does not satisfy INDEC 0 .

Thus, once we have proven Theorem 4.1 it will follow that $A B W_{0}, G_{\delta} A B W_{0}$, $\mathrm{F}_{\sigma \delta} \mathrm{ABW}_{0}$, and $\mathrm{G}_{\delta \sigma} \mathrm{ABW}_{0}$ are incomparable with both $\Delta_{1}^{1}-\mathrm{CA}_{0}$ and INDEC .

To prove Theorem 4.1, we will show that van Wesep's model $\mathcal{N}$ in Nee, Section 3], $\mathrm{vW}$, is indeed a model of $A B W_{0}$. Neeman Nee has already shown that $\mathcal{N}$ is a model of $\neg \mathrm{INDEC}_{0}$. The key to showing that $\mathcal{N}$ satisfies $A B W_{0}$ is to prove a modified version of $\mathrm{vW}$, Lemma 1.5, Sublemma 1], which Neeman describes in [Nee, Lemma 3.6].

Van Wesep's forcing conditions are similar to the ones that we defined in the previous section, with one main difference. Rather than tagging with ordinals, VW] tags his trees with elements of a fixed computable linear order with no infinite hyperarithmetic descending sequences. Fix a nonstandard initial segment of such a (computable) linear ordering (for example, one could use the Harrison linear ordering [Har68), and call it $\gamma\left(\mathrm{vW}\right.$ refers to it as $\left.I_{a}\right)$.

Our forcing conditions are similar to those of [Nee, $\mathrm{vW}$ ]; they are triplets $\left\langle T^{p}, f^{p}\right.$, $\left.h^{p}\right\rangle$ such that

(1) $T^{p} \subset \omega^{<\omega}$ is a finite tree.

(2) $f^{p}: \omega \rightarrow T^{p}$ is such that $\operatorname{dom}\left(f^{p}\right) \subset_{f} \omega$. 
(3) $h^{p}: T^{p} \rightarrow \gamma \cup\{\infty\}$ so that

(a) $\left(\forall \sigma, \tau \in T^{p}\right)\left[\sigma \subset \tau \Rightarrow h^{p}(\sigma)>_{\gamma} h^{p}(\tau)\right]$,

(b) $\left(\forall \sigma \in T^{p}\right)\left[\left((\exists i) \sigma \subseteq f^{p}(i)\right) \Rightarrow h^{p}(\sigma)=\infty\right]$, and

(c) $h^{p}(\emptyset)=\infty$.

By fiat, $\infty>\infty$ and $\infty>\omega_{1}^{C K}$. Let $P$ denote the set of the above forcing conditions. We note that in $\left[\mathrm{vW}\right.$, instead of tagging nodes of $T^{p}$ with $\infty$, the author simply leaves these nodes untagged, and so $h^{p}$ is only defined on the set of nodes in $T^{p}$ that are not extended by $f^{p}(i), i \in \operatorname{dom}\left(f^{p}\right) \subset_{f} \omega$.

For $p, q \in P, \mathrm{vW}]$ defines $p \leq q$ if and only if

(4) $T^{q} \subseteq T^{p}$,

(5) (a) $\operatorname{dom}\left(f^{q}\right) \subseteq \operatorname{dom}\left(f^{p}\right)$,

(b) $\left(\forall i \in \operatorname{dom}\left(f^{q}\right)\right)\left[f^{q}(i) \subseteq f^{p}(i)\right]$,

(6) $h^{q}=h^{p} \uparrow T^{q}$.

Note that we have eliminated conditions $(5 \mathrm{c})$ and $(5 \mathrm{~d})$ from Section 3.2 . Our proof that $\mathcal{N} \vDash \mathrm{ABW}_{0}$ will depend upon this fact (Lemma 4.6 below). Let $\mathbb{P}=\langle P, \leq\rangle$ and $G$ be any sufficiently $\mathbb{P}$-generic filter.

Another difference between the construction of van Wesep's model, $\mathcal{N}$, in [Nee, $\mathrm{vW}$ ] and our construction of $\mathcal{M}_{\infty}$ in Section 3 is that $\mathcal{N}$ is of the form

$$
N=\bigcup_{\substack{F \subset f_{f} \\ F \subseteq S^{*}}} M_{F}^{G},
$$

where $M_{F}^{G}$ is defined analogously to our notion of $M_{F}$ in Section 3 but $S^{*} \subseteq \omega$ is such that

$$
(\forall i, j \in \omega)\left[\left(f_{i}^{G}(0)=f_{j}^{G}(0)\right) \Rightarrow\left(i \in S^{*} \Leftrightarrow j \in S^{*}\right)\right]
$$

and $f_{i}^{G}=\bigcup_{p \in G} f^{p}(i), i \in \omega$ (analogous to the definition of $\alpha_{i}^{G}$ in Section 3). Note that (11) above is a consequence of the (more complicated) definition of $S^{*}$ given in $\mathrm{vW}$. In $\mathrm{vW}$, van Wesep uses the definition of $S^{*}$ and the construction of $\mathcal{N}$ to show that $\mathcal{N} \not \models \Delta_{1}^{1}-\mathrm{CA}_{0}$, while, in [Nee, Neeman uses these facts to conclude that $\mathcal{N} \not \models$ INDEC 0 .

For every finite $F \subset_{f} \omega$, we construct $M_{F}^{G}$ analogously to our construction of $M_{F}$ in Section 3 . The construction of $M_{F}^{G}$ given by [Nee, $\mathrm{vW}$ ] is different from, but equivalent to our construction of $M_{F}^{G}$. In particular, [Nee, $\sqrt{\mathrm{vW}}$ ] construct $M_{F}^{G}$ in $L_{\omega_{1}^{C K}}\left(T, f_{i}^{G}: i \in F\right)$, while our construction lives in $\mathcal{P}(\omega)$. However, since the elements $T^{G}$ and $f_{i}^{G}, i \in \omega$, are generic, it follows that $\omega_{1}^{C K}$ relative to $T, f_{i}^{G}, i \in F$, is equal to $\omega_{1}^{C K}$ (relative to $\emptyset$ ), and we therefore have that

$$
M_{F}^{G}=\mathcal{P}(\omega) \cap L_{\omega_{1}^{G K}}\left(\left\{T^{G}\right\} \cup\left\{f_{i}^{G}: i \in F\right\}\right),
$$

where $M_{F}^{G}$ is constructed analogously to $M_{F}$ in Section 3 of this article. Furthermore, for every $F \subset_{f} \omega$, we have $\left[T^{G}\right] \cap M_{F}^{G}=\left\{f_{i}^{G}: i \in F\right\}, M_{F}^{G}=\operatorname{HYP}\left(T^{G}, f_{i}^{G}\right.$ : $i \in F$ ), and $\mathcal{M}_{F} \vDash \Sigma_{1}^{1}-\mathrm{AC}_{0}$ (see [Nee, $\mathrm{vW}$ ] for more details).

Van Wesep [vW, Definition 1.2] defines retaggings (or absolute reducts, as he calls them) exactly as we do in Definition 3.2 above. Using the genericity of $G \subseteq \mathbb{P}$ and retaggings, one can prove the following theorem vW, Lemma 1.5, Sublemma 2 ], which we will use in our proof of Theorem 4.7 below to show that $\mathcal{N} \vDash A B W_{0}$. The main idea behind the proof of $\mathrm{vW}$, Lemma 1.5, Sublemma 2] is similar to that 
of Theorem 3.17 above, or [Mon, Lemma 2.14]. We give only but a sketch of the proof; for more details we ask the reader to consult vW, Lemma 1.5, Sublemma $2]$.

Lemma $4.3\left(\sqrt{\mathrm{vW}}\right.$, Lemma 1.5, Sublemma 2]). For any $I, J \subset_{f} \omega$, we have that

$$
M_{I}^{G} \cap M_{J}^{G}=M_{I \cap J}^{G} .
$$

Proof. (Sketch) By our construction of $M_{F}^{G}, F \subset_{f} \omega$, above, it is clear that $M_{I \cap J}^{G} \subseteq$ $M_{I}^{G} \cap M_{J}^{G}$. Therefore, it suffices to prove that $M_{I}^{G} \cap M_{J}^{G} \subseteq M_{I \cap J}^{G}$. In other words, we will show that if $X \in \omega^{\omega}$ satisfies $X \in M_{I}^{G}$ and $X \in M_{J}^{G}$, then we also have that $X \in M_{I \cap J}^{G}$.

Let $X \in M_{I}^{G} \cap M_{J}^{G}$. Let $F=I \cap J$, and $H, G \subset_{f} \omega$ be such that $F \cap H=$ $F \cap G=\emptyset, I=F \cup H, J=F \cup G$. Let $\nu<\omega_{1}^{C K}$ be such that there exist $e_{0}, e_{1} \in \omega$ such that

$$
X=S_{\nu, I, e_{0}}=S_{\nu, J, e_{1}}
$$

(where $S_{\mu, A, z}, \mu<\omega_{1}^{C K}, A \subset_{f} \omega, z \in \omega$, is as defined in Section 3.1). The proof rests on the following proposition, which we state, but will not prove (for a proof, consult VW, Lemma 1.5, Sublemma 2]). As we have already remarked, the main idea behind the proof of the following proposition can be found in the proof of Theorem 3.17 above, or [Mon, Lemma 2.14].

Claim 4.4. Let $p \in \mathbb{P}$ be such that

$$
p \Vdash \boldsymbol{X}=\boldsymbol{S}_{\nu, I, e_{0}}=\boldsymbol{S}_{\nu, J, e_{1}} .
$$

Then, for all $n \in \omega$, we have that $n \in X$ if and only if there exists $q \leq p$ such that

(1) $q \Vdash \boldsymbol{n} \in \boldsymbol{S}_{\nu, I, e_{0}}$.

(2) $T^{q} \subseteq T^{G}$.

(3) $h^{q}$ is $\omega \nu+\omega$-good for $T^{G}$ (see Definition 3.16 above).

(4) For all $i \in F$, we have that $f^{q}(i)$ is the longest initial segment of $f_{i}^{G} \in\left[T^{G}\right] \subseteq \omega^{\omega}$ on $T^{q}$.

The proposition implies that $X$ is hyperarithmetic in $T^{G} \oplus \bigoplus_{i \in F} f_{i}^{G}$, and hence $X \in M_{F}^{G}=M_{I \cap J}^{G}$, as required.

We now state $\mathrm{vW}$ Lemma 1.5, Sublemma 1] the main idea which can be found in [Nee, Lemma 3.6].

Lemma 4.5 ( $\sqrt{\mathrm{vW}}$, Lemma 1.5, Sublemma 1). Suppose that $F \subseteq I \subset_{f} \omega, Z_{0} \in$ $M_{F}^{G}, X_{0} \in M_{I}^{G}$, and that $B(X, Z)$ is an arithmetical predicate with only the free variables shown, such that $B\left(X_{0}, Z_{0}\right)$ holds. Then, there exists $J \subset_{f} \omega, X_{1} \in M_{J}^{G}$, such that $I \cap J=F$ and $B\left(X_{1}, Z_{0}\right)$ holds. Moreover, for every $j \in J$ there exists $i \in I$ such that $f_{i}^{G}$ and $f_{j}^{G}$ have a nontrivial initial segment in common. Hence, by (11) above, $I \subseteq S^{*} \Rightarrow J \subseteq S^{*}$.

Next, we will prove a modified version of [vW, Lemma 1.5, Sublemma 1] to show that, indeed, $\mathcal{N} \vDash A B W_{0}$. The main idea of our proof is derived from that of van Wesep. Van Wesep's proof depends upon automorphisms of $\mathbb{P}$, which we defined in Section 3.6 Note that, via the same proof, Lemma 3.9 also holds for $\mathbb{P}$ (in place of $\boldsymbol{P})$. 
Lemma 4.6. Suppose that $F \subseteq I \subset_{f} \omega, Z_{0} \in M_{F}^{G}, X_{0} \in M_{I}^{G}$, and $B(X, Z)$ is an arithmetical predicate with only the free variables shown, such that $B\left(X_{0}, Z_{0}\right)$ holds. Then, for any given $k \in \omega$, there exists $J_{k} \subset_{f} \omega, X_{k} \in M_{J_{k}}^{G}$, such that $I \cap J_{k}=F$, $B\left(X_{k}, Z_{0}\right)$ holds, and

$$
X_{0}\left\lceil k=X_{k} \uparrow k .\right.
$$

Moreover, for every $j \in J_{k}$ there exists $i \in I$ such that $f^{G}(i)$ and $f^{G}(j)$ have a nontrivial initial segment in common. Therefore, by (1) above, $I \subseteq S^{*} \Rightarrow J_{k} \subseteq S^{*}$.

Proof. The proof of Lemma 4.6 resembles that of $\mathrm{vW}$, Lemma 1.5, Sublemma 1].

Let $I=F \cup H_{1}, H_{1} \cap F=\emptyset$, and $k \in \omega$ be given. Suppose that $Z_{0}=S_{\nu_{0}, F, e_{0}}$ and $X_{0}=S_{\nu_{1}, I, e_{1}}, \nu_{0}, \nu_{1}<\omega_{1}^{C K}, F \subseteq I \subset_{f} \omega, e_{0}, e_{1} \in \omega$. Now, let $\sigma_{k} \in \omega^{<\omega}$ be the initial segment of $X_{0} \in \omega^{\omega}$ of length $k$, and $p \in G$ be such that

$$
p \Vdash B\left(\boldsymbol{S}_{\nu_{1}, I, e_{1}}, \boldsymbol{S}_{\nu_{0}, F, e_{0}}\right) \wedge\left(\boldsymbol{\sigma}_{k} \subset \boldsymbol{S}_{\nu_{1}, I, e_{1}}\right) .
$$

Finally, let $H_{2} \subset_{f} \omega$ be such that $H_{2} \cap\left(F \cup H_{1}\right)=\emptyset$, and $H_{0}=H_{1} \cup H_{2}$ satisfies $\operatorname{dom}\left(f^{p}\right) \subseteq F \cup H_{0}$.

As in [vW, Lemma 1.5, Sublemma 1], we call $q \in \mathbb{P}$ a doublet if there exists $n \in \omega$ such that $\operatorname{dom}\left(f^{q}\right)=2 n$ and for all $m<n$ we have that $f^{q}(m)=f^{q}(m+n)$. Call $n$ the period of the doublet, and note that for any $n \in \omega$, the set of doublets of period $>n$ is dense in $\mathbb{P}$. Let $n_{1} \in \omega$ be such that $n_{1}>\max \left\{F \cup H_{0}\right\}$, and let $q \in G$ be such that $q \leq p$ and $q$ is a doublet with period $n>n_{1}, n \in \omega$. Let $L_{i}=\left\{l \in \omega:\left(\exists j \in \bar{H}_{i}\right)[l=j+n]\right\}$, for $i=0,1,2$, and note that, by the construction of $L_{i}(i=0,1,2)$ we have that $L_{0}=L_{1} \cup L_{2}$ and $L_{0} \cap\left(F \cup H_{0}\right)=\emptyset$.

Now, let $r \geq q$ be identical to $p \in \mathbb{P}$, except that

(i) $\operatorname{dom}\left(f^{r}\right) \subseteq F \cup L_{0}$.

(ii) $i \in \operatorname{dom}\left(\bar{f}^{r}\right) \Leftrightarrow\left[\left(i \in F \cap \operatorname{dom}\left(f^{p}\right)\right) \vee\left(i-n \in H_{0} \cap \operatorname{dom}\left(f^{p}\right)\right)\right]$.

(iii) $f^{r}(i)=f^{p}(i)$, for all $i \in F \cap \operatorname{dom}\left(f^{p}\right)$, and $f^{r}(i+n)=f^{p}(i)$, for all $i \in H_{0}$.

In constructing $r \geq q$ above, we have simply translated the part of the domain of $f^{p}$ that is $H_{0} \subseteq \operatorname{dom}\left(f^{p}\right)$ to the right by $n$ and onto $L_{0} \subseteq \operatorname{dom}\left(f^{r}\right)$. Furthermore, since $r \geq q$ and $q \in G$, we have that $r \in G$.

Note that, by our construction of $r \geq q$, there is an automorphism of $\mathbb{P}$ taking $p$ to $r$, and so, via a suitably modified version of Lemma 3.9 (i.e. the automorphism lemma), we have that

$$
r \Vdash B\left(\boldsymbol{S}_{\nu_{1}, F \cup L_{1}, e_{1}}, \boldsymbol{S}_{\nu_{0}, F, e_{0}}\right) \wedge\left(\boldsymbol{\sigma}_{k} \subset \boldsymbol{S}_{\nu_{1}, F \cup L_{1}, e_{1}}\right) .
$$

Therefore, if we set

$$
X_{k}=S_{\nu_{1}, J_{k}, e_{1}} \in \omega^{\omega} \text { and } J_{k}=F \cup L_{1} \subset_{f} \omega,
$$

then it follows that $X_{k} \in M_{J_{k}}^{G}$ satisfies the conclusion of Lemma 4.6.

We are now ready to show that van Wesep's $\mathcal{N}$ is indeed a model of $A B W_{0}$.

Theorem 4.7.

$$
\mathcal{N} \vDash \mathrm{ABW}_{0} .
$$

Proof. Let $A(X)$ be a bounded arithmetic predicate with parameters from $N=$ $\bigcup_{F \subset_{f} S^{*}} M_{F}^{G}$, and such that $A(X)$ has infinitely many solutions $X \in N$. By definition of $N$, there exists $F \subset_{f} \omega$ such that $A(X)$ is an arithmetic predicate with parameters from $M_{F}^{G}$. 
There are two cases to consider. The first case says that every solution of $A(X)$ lies in $M_{F}^{G}$. In this case we use the fact that $M_{F}^{G}=\operatorname{HYP}\left(T^{G}, f_{i}^{p}: i \in F\right)$ to conclude that $\mathcal{M}_{F} \vDash \Sigma_{1}^{1}-\mathrm{AC}_{0}$, and thus, by Theorem 2.1, $\mathcal{M}_{F} \vDash A B W_{0}$. Therefore, there exists $X \in M_{F}^{G} \subseteq N$ such that $X$ is an accumulation point for the set $\left\{Z \in 2^{\omega}: A(Z)\right\} \cap N$, from which it follows that $\mathcal{N} \vDash \mathrm{ABW}_{0}$.

The second case says that there exists $X \in N$ such that $X \notin M_{F}^{G}$ and $A(X)$ holds. In this case it follows from Lemmas 4.3 and 4.6 above that for every $k \in \omega$ there exists $Y \in N$ such that $Y \neq X$, but $Y\lceil n=X \uparrow n$. Hence, $X \in N$ is an accumulation point for the set $\left\{Z \in 2^{\omega}: A(Z)\right\} \cap N$, and therefore we have that $\mathcal{N} \vDash \mathrm{ABW}_{0}$.

\section{REFERENCES}

[AK] C.J. Ash and J.F. Knight, Computable structures and the hyperarithmetical heirarchy, North-Holland Publishing Co., Amsterdam, 2000. MR.1767842 (2001k:03090)

[Fri] H. Friedman, Subsystems of set theory and analysis, Ph.D. thesis, MIT, 1967.

[Fri75] - Some systems of second order arithmetic and their use, Proc. Int. Congr. Math., Vancouver, B.C. (1974), vol. 1, Canadian Mathematical Congress, Montreal, Quebec, 1975, pp. 235-242. MR0429508 (55:2521)

[Har68] J. Harrison, Recursive pseudo-well-orderings, Transactions of the American Mathematical Society 131 (1968), 526-543. MR0244049 (39:5366)

[Jul] P. Jullien, Contribution à l'étude des types d'ordre dispersés, Ph.D. Thesis. Marseille (1969).

[Kre62] G. Kreisel, The axiom of choice and the class of hyperarithmetic functions, Indag. Math. 24 (1962), 307-319. MR0140418 (25:3838)

[Mon] A. Montalbán, On the $\Pi_{1}^{1}$-separation principle, Mathematical Logic Quarterly 54 (2008), 563-578. MR2472464 (2009j:03016)

[Mon06] A. Montalbán, Indecomposable linear orderings and hyperarithmetic analysis, J. Math. Log. 6 (2006), 89-120. MR2250955 (2007e:03102)

[Nee] I. Neeman, The strength of Jullien's indecomposability theorem, J. Math. Log. 8 (2008), 93-119. MR2674002

[Sim] S.G. Simpson, Subsystems of second order arithmetic, Springer-Verlag, 1999. MR1723993(2001i:03126)

[Soa] R.I. Soare, Recursively enumerable sets and degrees, Springer-Verlag, 1987. MR882921 (88m:03003)

[Spe55] C. Spector, Recursive well-orderings, J. Symbolic Logic 20 (1955), 151-163. MR0074347 $(17: 570 \mathrm{~b})$

[Ste78] J.R. Steel, Forcing with tagged trees, Ann. Math. Log. 15 (1978), 55-74. MR.511943 (81c:03044)

[vW] R.A. van Wesep, Subsystems of second order arithmetic, and descriptive set theory under the axiom of determinateness, Ph.D. Thesis, Berkeley, 1977.

Department of Pure Mathematics, University of Waterloo, Waterloo, Ontario, CANADA N2L 3G1

Current address: Department of Mathematics, Vanderbilt University, Nashville, Tennessee 37240 